\title{
Generally Covariant Quantum Field Theory and Scaling Limits
}

\author{
Klaus Fredenhagen and Rudolf Haag \\ II. Institut für Theoretische Physik der Universität Hamburg, Luruper Chaussee 149, D-2000 Hamburg \\ 50, Federal Republic of Germany
}

\begin{abstract}
The formulation of a generally covariant quantum field theory is described. It demands the elimination of global features and a characterization of the theory in terms of the allowed germs of families of states. A simple application is the computation of counting rates of accelerated idealized detectors. As a first orientation we discuss here the consequences of the assumption that the states have a short distance scaling limit. The scaling limit at a point gives a reduction of the theory to tangent space. It contains kinematical information but not the full dynamical laws. The reduced theory will, under rather general conditions, be invariant under translations and under a proper subgroup of the linear transformations in tangent space. One interesting possibility is that it is invariant under SLR(4). Then the macroscopic metric must evolve as a cooperative effect in finite size regions. The other natural possibility is that each family (coherent folium) of states defines a microscopic metric by the scaling limit and the tangent space theory reduces to a theory of free massless fields in a Minkowski space. Irrespective of the assumption of a scaling limit we show that the theory can be constructed from strictly local information.
\end{abstract}

\section{The Physical Picture}

Quantum Field Theory may be regarded as a synthesis of special relativity (incorporating the restricted principle of locality) and quantum theory. In classical physics the principle of locality, originating in the development of electro-dynamics by Faraday and Maxwell, was sharpened in special relativity theory by the statement that "no causal influence can propagate faster than light" and was ultimately implemented in the general theory of relativity in its most stringent form: The laws of nature regulate only the behavior of physical quantities in the infinitesimal neighborhood of each point.

In special relativity there is still one last remnant of global laws, namely the rigid metric structure of space-time. This is removed in the general relativity theory in the following way:

(i) The basic physical quantities relate to the tangent spaces at the points of the 4- 
dimensional space-time continuum $M$, i.e. they are vector fields, tensor fields etc. Among them is the tensor field $g_{\mu \nu}$ governing the metric.

(ii) The laws are differential equations for these quantities (field equation).

(iii) The field equations are required to be intrinsic, i.e. independent of the coordinate system used to describe $M$. This last requirement, also called "general covariance" is extremely restrictive once the types of all fields with which one has to deal are specified.

It is by no means clear that Quantum Theory should (or even can) follow the development of classical theory towards an elimination of global features from the formulation of basic laws. In the last decade quantum field theory has relied increasingly on the use of the Feynman path integral to compute a distinguished global state. In addition there is justified doubt that the notion of a space-time continuum (or in fact any continuum) makes physical sense in the small. Nevertheless it seems important to us to clarify whether it is possible to incorporate the unrestricted principle of locality and general covariance into quantum theory. It is the purpose of the present paper to study how this can be achieved.

Let us, first, consider general covariance and ask whether it can be interpreted in an active sense as invariance of the laws under a very large symmetry group, the group of local diffeomorphisms of the manifold. Throughout the paper we shall only be concerned with local properties. Suppose we have a chart mapping some (open, contractible) region of $\mathscr{M}$ into $\mathcal{O}_{0} \subset \mathbb{R}^{4}$, the coordinates being denoted by $x=\left\{x^{\mu}\right\}$, $\mu=0,1,2,3$. Consider a smooth, invertible transformation

$$
x \rightarrow x^{\prime}(x)
$$

such that there is a closed subregion $\mathscr{K} \subset \mathcal{O}_{0}$ which is transformed into itself as a set and so that (1) is the identity transformation outside of $K$ :

$$
\text { for } \quad x \in \mathscr{K} \quad x^{\prime} \in \mathscr{K} \quad \text { and for } \quad x \in \mathcal{O}_{0} \backslash \mathscr{K} \quad x^{\prime}=x .
$$

We may interpret this transformation either as a transition to another chart so that $x$ and $x^{\prime}$ represent the same point of $\mathscr{M}$ in two different coordinate systems ("passive interpretation") or as a shifting of the points of the manifold so that $x^{\prime}$ describes the image point in the same chart. In this second, "active" interpretation (1) describes a local diffeomorphism of $\mathscr{M}$. Clearly, if in the classical theory we have a field configuration allowed by the laws, the action of a diffeomorphism on it is well defined (because the diffeomorphism of $\mathscr{M}$ defines also maps of the tangent spaces) and there results a different field configuration on the manifold which again satisfies the laws. If, metaphysically, we imagine that the points of the manifold have a reality in their own right, then the transformed field configuration is a different physical situation from the original one. This, however, would mean that we have no deterministic prediction of the future from the past because a local diffeomorphism changes the fields in the region where it acts nontrivially while leaving them unchanged in the past where it acts trivially. To understand the significance of this let us consider the case of generally covariant electrodynamics. We have then only the electromagnetic field $F_{\mu \nu}$ and the metric field $g_{\mu \nu}$ satisfying together the Maxwell-Einstein equations. Suppose these fields are known prior to some space- 
like surface $\Sigma$ then, indeed, without conditions for the coordinatization of future points one has no Cauchy problem. As is well known, the Einstein equations give four constraints for the metric field and its normal derivative on the surface $\Sigma$ and only 6 "dynamical" equations for the 10 quantities $g_{\mu v}$. If the observer establishes the labelling of future points of $M$ (coordinatization) by means of conventions using only $F_{\mu \nu}$ and $g_{\mu \nu}$, then the mentioned diffeomorphism will have no effect on his observations. The "change of the physical situation" is exactly compensated by his change of the labelling of the points. The field equations together with such coordinate conditions provide a deterministic scheme. The active interpretation of general covariance demands therefore that we imagine the possibility of decoupling the labelling of (future) points of the manifold from the prevailing configurations of fields which obey a closed system of intrinsic field equations. It need not concern us here whether such an imagination fits into the ideal of a complete, classical field theory of the world ${ }^{1}$. On the one hand there are circumstances in which such a decoupling is approximately realizable. And there is quantum physics which, in its present formulation, starts from a cut between the macroscopic world and the quantum phenomena.

In orthodox quantum theory we have a division of the world into a macroscopic, naively described part associated with "the observer" and the microscopic regime behind it. The need for such a division is stressed in Bohr's words that we have to be able "to tell our friends what we have done and what we have learned ${ }^{2}$." Space-time, as attached to the observer establishing a reference frame by means of "rigid" bodies, clocks, signals is a concept of the macroscopic world serving as a vessel in which the phenomena are placed. It is inherent in this picture that this macroscopic space-time continuum and its coordinatization by the observer is prior to the quantum events studied and unaffected by them. Since our direct means of determining the placement of a phenomenon in this frame is limited to the rather poor accuracy of, say, $10^{-7} \mathrm{~cm}$, the structural assumptions and detailed formalism of the theory must be judged indirectly by their ability to account correctly for the observed phenomena. Among these assumptions is the mathematical representation of physical quantities as elements of a noncommutative algebra and, in quantum field theory, the generating of this algebra from quantities associated with arbitrarily small space-time regions. The spectacular quantitative success of this formalism in Quantum Electrodynamics and its qualitative success in elementary particle physics, using significantly subdivisions of space-time down to extensions of $10^{-16} \mathrm{~cm}$ suggests that it is worthwhile to pursue this approach till its limits become (clearly) apparent.

\footnotetext{
1 The realistic interpretation of the space-time points underlines their rôle as the carrier of physical action as envisaged by Faraday. For that reason Einstein sometimes advocated the reintroduction of the term "ether" e.g. in [1]. On the other hand it contrasts with the point of view, probably first advocated by Leibniz and shared by many to-day, that space-time is just an ordering device for physical events without independent significance

2 This description must be unambiguous and objective ("intersubjective") and this is probably the reason why it may still use concepts and laws of classical physics to shorten the explanation. The distinction between macroscopic and microscopic is necessary but not sufficient in the division as exemplified by the existence of macroscopic quantum phenomena
} 
The general frame indicated is then: We consider a (classical) 4-dimensional manifold $\mathscr{M}$ and associate to each open region $\mathcal{O} \subset \mathscr{M}$ an involutive algebra $\mathfrak{H}(\mathcal{O})^{3}$. The selfadjoint elements of $\mathfrak{U}(\mathcal{O})$ are usually interpreted as the "observables" which can be measured in $\mathcal{O}$. This becomes somewhat painful if we think of regions of $10^{-16} \mathrm{~cm}$ diameter, since it suggests a rather incredible employment of hardware which does not correspond to actual laboratory practice. A better picture is perhaps to maintain that certain positive elements in $\mathfrak{U}(\mathcal{O})$ represent elementary events whose intrinsic extension and placement in $\mathscr{M}$ is described by $\mathcal{O}$. Although in $\mathscr{M}$, as the macroscopic space-time of an observer, there is a classical, pseudo-Riemannian metric available we should not use this metric to formulate the microscopic laws. It has to be taken into account as a consistency condition matching prevailing quantum states with the classical description of space-time by the observer. In order to accommodate general covariance and unrestricted locality, the algebra $\mathfrak{A}$ has to be flexible, free of relations between quantities associated with disjoint space-time regions. In this respect we must depart from the usual scheme of Quantum Field Theory in Minkowski space, where we have a natural correspondence between the causal independence of two distant regions and the commutativity of observables in these regions. H. Ekstein had advocated for many years that one should distinguish between "observables" and "observation procedures," the former being equivalence classes of the latter, and he pointed out that this distinction becomes crucial in the generally covariant extension of quantum field theory $[2,3]$. We are ready to follow his advice now.

In the notion of "equivalence classes of procedures," i.e., relations between algebraic quantities of different regions, the concept of "state" enters. A state $\omega$, intuitively understood as summarizing previous history-or our knowledge of it as far as it is relevant - defines a probability for subsequent events. It is mathematically described by a positive linear form (expectation functional) on $\mathfrak{A}$. Given a state $\omega$ we obtain in a canonical manner a representation $\pi_{\omega}$ of $\mathfrak{U}$ by an operator algebra in a Hilbert space $\mathfrak{H}_{\omega}$ (Gelfand-Naimark-Segal construction), we get a full folium $\mathscr{F}_{\omega}$ of related states, namely those represented by vectors and density matrices of $\mathfrak{H}_{\omega}$; furthermore we (may) get relations in the algebra. Two algebraic elements $A_{1}, A_{2}$ are equivalent with respect to this folium of states if $\omega^{\prime}\left(B^{*} B\right)=0$ for all $\omega^{\prime} \in \mathfrak{F}_{\omega}$ (putting $A_{1}-A_{2}=B$ ). The set of such relations defines a 2-sided ideal $J_{\omega}(\mathcal{O})$ in every subalgebra $\mathfrak{A}(\mathcal{O})$; it is the kernel of the representation $\pi_{\omega}$, i.e. the set of elements in $\mathfrak{U}(\mathcal{O})$ which are represented by the zero operator in $\mathfrak{H}_{\omega}$. For mathematical convenience we may pass from the operator algebras $\pi_{\omega}(\mathfrak{A}(\mathcal{O}))$ to the associated von Neumann algebras $\mathfrak{R}_{\omega}(\mathcal{O})$ in the Hilbert space $\mathfrak{H}_{\omega} \cdot{ }^{4}$

3 An algebra over the complex numbers with an involution (adjoint operation, *-operation) denoted by $A \rightarrow A^{*}$. At this stage we leave open the topology put on those algebras. They may be thought of as $C^{*}$ algebras or as equipped with a set of seminorms. The correspondence $\mathcal{O} \rightarrow \mathfrak{U}(\mathcal{O})$ will be called the net of local algebras. Of course $\mathfrak{A}\left(\mathcal{O}_{1}\right) \subset \mathfrak{A}\left(\mathcal{O}_{2}\right)$ if $\mathcal{O}_{1} \subset \mathcal{O}_{2}$ and all algebras shall have a common unit element. We shall sometimes write $\mathfrak{A}$ for the algebra of the largest region under consideration

4 Typically there is no norm on $\mathfrak{A}$ satisfying the condition $\|A B\| \leqq\|A\|\|B\|$, and therefore the representors $\pi_{\omega}(A)$ may be unbounded operators. Therefore the transition from $\pi_{\omega}(\mathfrak{Q})$ to $\mathfrak{R}_{\omega}$ needs some explanation. There is, however, a canonical procedure which is described in the appendix. For an abstract construction of Banach algebras associated with the Borchers algebra, see [13] 
The first step in the formulation of a specific theory is a description of the net of algebras $\mathfrak{X}(\mathcal{O})$. The second is the characterization of all possible states, i.e. all physically allowed positive linear forms on $\mathfrak{U}$. The division between these two tasks is to some degree movable. The less structure one incorporates in the specification of $\mathfrak{A}$ the more restrictive information must be put into the second. In the present paper we take the simplest possible choice for $\mathfrak{U}$, the free tensor algebra of smooth scalar functions on $\mathscr{M}$. We shall sometimes refer to it as the Borchers algebra [4] of a scalar field, although in contradistinction to [4] it is not equipped with relations expressing local commutativity. It will be described explicitly at the beginning of Sect. $\mathrm{II}^{5}$. A local diffeomorphism of $\mathscr{M}$ induces an automorphism of $\mathfrak{A}$. That this action is not merely an empty definition but carries physical content is illustrated in Sect. II. It allows us to compare the counting rates of idealized detectors in different (accelerated) motions.

Concerning the specification of the set of physically admissible states, we make here several tentative assumptions of a general nature. The first is that there are states which are "everywhere primary." These are such that the von Neumann algebras $\mathfrak{R}_{\omega}(\mathcal{O})$ have only trivial center for each sufficiently small, contractible region $\mathcal{O}$ (they are "factors") ${ }^{6}$. All other admissible states shall be built up by convex combinations (possibly continuous mixing) from such primary states. We can then restrict attention to these primary states and folia. We denote the set of admissible states in this (primary) class over an algebra $\mathfrak{X}(\mathcal{O})$ by $\mathscr{S}(\mathcal{O})$, the set of such folia by $\mathscr{F}(\mathcal{O})$. Since $\mathfrak{U}\left(\mathcal{O}_{1}\right) \subset \mathfrak{U}\left(\mathcal{O}_{2}\right)$ for $\mathcal{O}_{1} \subset \mathcal{O}_{2}$ and since the states considered are assumed to be primary over all regions, we have natural restriction maps $\mathscr{S}\left(\mathcal{O}_{2}\right) \rightarrow \mathscr{S}\left(\mathcal{O}_{1}\right)$, $\mathscr{F}\left(\mathcal{O}_{2}\right) \rightarrow \mathscr{F}\left(\mathscr{O}_{1}\right)$. Mathematically, $\mathscr{S}$ and $\mathscr{F}$ are presheafs. This allows us to define a "germ" in $\mathscr{S}$ or $\mathscr{F}$ at a point $P \in \mathscr{M}$ as an equivalence class consisting of all states respectively folia such that there exists some open neighborhood of $P$ on which their restrictions coincide. We assume further that there remain no quantum observables at a point: $\cap \mathscr{R}_{\omega}(\mathcal{O})=\{\lambda 1\}$, where the intersection is taken over all neighborhoods of a point $P \in \mathscr{M}$. This implies that two states in the same folium become indistinguishable in infinitesimally small regions; more precisely, the norm distance of their restriction to algebras $\mathfrak{U}\left(\mathcal{O}_{n}\right)$ goes to zero as $\mathcal{O}_{n}$ shrinks to a point. This generalizes the principle of "local definiteness" stated in [5]. There the metric structure was assumed to the given, and as a consequence a unique germ was assumed. Now we have to deal with a stalk of allowed germs.

As a first orientation towards a description of these germs we use in Sect. III a scaling assumption proposed in [5] which reduces the theory to one in the tangent

5 The generalization to fields of vector, tensor-character is obvious. Spinor fields and gauge connections require a more subtle starting point. The essential structure used is that if $\mathcal{O}=\bigcup_{i} \mathcal{O}_{i}$ (open covering), then $\mathfrak{H}(\mathcal{O})$ shall be the free algebra generated from the $\mathfrak{A}\left(\mathcal{O}_{i}\right)$, for which we use the symbol $\mathfrak{A}(\mathcal{O})=\vee \mathfrak{A}\left(\mathcal{O}_{i}\right)$. By

"free" we mean that no new relations are introduced, a typical element of $\mathfrak{A}\left(\mathcal{O}_{1}\right) \vee \mathfrak{A}\left(\mathcal{O}_{2}\right)$ is a sum of products of factors from $\mathfrak{A}\left(\mathcal{O}_{1}\right)$ and $\mathfrak{U}\left(\mathcal{O}_{2}\right)$, where products composed of the same factors appearing in different order are considered as different elements (apart from the identification of elements in the intersection region $\mathcal{O}_{1} \cap \mathcal{O}_{2}$ and relations within the algebras $\left.\mathfrak{A}\left(\mathcal{O}_{1}\right), \mathfrak{A}\left(\mathcal{O}_{2}\right)\right)$

6 This is suggested by our knowledge of customary theories in Minkowski space, but there may be reasons for abandoning it ultimately 
space at a point, and yields for each primary folium $\mathscr{F}$ and each point $P$ one distinguished state $\omega_{P}^{\mathscr{F}}$ for the tangent space theory. The set $\left\{\omega_{P}^{\mathscr{F}}\right\}$ forms a fiber bundle over $\mathscr{M}$. The primary folia give sections in this bundle. The scaling limit carries, of course, much less information than the above mentioned germs. The reduced state $\omega_{P}^{\mathscr{F}}$ is dilation covariant by construction, translation invariant by reasonable continuity assumptions. Due to general covariance the action of the full linear group in tangent space is defined on the fibers. If the $\omega_{P}^{\mathscr{F}}$ were not invariant under some subgroup of GLR(4) each primary folium would define a flat affine connection in $\mathscr{M}$. To allow curvature there must be invariance under some subgroup of GLR(4). The maximal possible invariance group is SLR(4). This may be an interesting possibility. If the scaling below the Planck length has physical meaning, the symmetry should be increased beyond Lorentz invariance. The macroscopic metric would then arise, analogous to the phase formation in statistical mechanics, as a symmetry breaking effect by cooperative action. The other natural possibility, suitable if one neglects the gravitational constant, is that the invariance group is isomorphic to the Lorentz group. Then a (primary) folium defines by the scaling limit a microscopic metric in the tangent space. In this case the most natural possibility is that the tangent space theory reduces to a free, massless quantum field theory in a Minkowski space and thereby defines the kinematical structure of the theory. The step from tangent space to infinitesimal neighborhoods (germs) will carry the dynamical information corresponding in the standard treatment to the field equations and the local part of commutation relations. There remains the problem of constructing the folia of extended regions from the germs. We shall address ourselves to this problem in Sect. IV.

\section{Idealized Detectors in Different Motion}

As mentioned above we take in this paper the simplest possible choice for $\mathfrak{A}$, the free tensor algebra of scalar test functions on the manifold. The elements of $\mathfrak{U}(\mathcal{O})$ are formal linear combinations of monomials $f^{(n)}$ of different degrees $n$ where $f^{(n)}=f^{(n)}\left(P_{1}, \ldots, P_{n}\right)$ is a complex valued smooth function on $\mathscr{M}^{n}=\mathscr{M} \times \mathscr{M} \times \cdots \mathscr{M}$ with support in $\mathcal{O}$ in every argument ${ }^{7}$. The algebraic product of $f^{(n)}$ with $g^{(n)}$ is just the tensor product

$$
f^{(n)} \cdot g^{(m)}=h^{(n+m)} ; \quad h^{(n+m)}\left(P_{1}, \ldots, P_{n+m}\right)=f^{(n)}\left(P_{1}, \ldots, P_{n}\right) g^{(m)}\left(P_{n+1}, \ldots, P_{n+m}\right) .
$$

The involution is the complex conjugation together with the inversion of the sequence of arguments. A diffeomorphism sending the point $P$ to $\chi P$ acts as the automorphism $\alpha_{\chi}$ on $\mathfrak{A}$ :

$$
\left(\alpha_{\chi} f^{(n)}\right)\left(P_{1}, \ldots, P_{n}\right)=f^{(n)}\left(\chi^{-1} P_{1}, \ldots, \chi^{-1} P_{n}\right) .
$$

A state $\omega$ on $\mathfrak{U}(\mathcal{O})$ is given by a hierarchy of distributions $\omega^{(n)} \in \mathscr{D}^{\prime}(\mathcal{O} \times \cdots \times \mathcal{O})$. $\omega^{(n)}\left(f^{(n)}\right)$ is the expectation value of the monomial element $f^{(n)}$ in the state $\omega$.

7 A more familiar notation where the "quantum field $\phi$ " is regarded as an "operator valued distribution" would be to write for the degree 1 elements $\phi\left(f^{(1)}\right)$ instead of $f^{(1)}$ 
The use of (2.2) to compute the effects of acceleration on detectors depends on our ability to assign an element in $\mathfrak{A}$ to a specified observation procedure. This can be done without essential ambiguity only under every idealized circumstances, i.e. when a purely geometric description of the procedure in space-time suffices. The simplest idealization is to neglect the spatial extension and internal structure and consider the detector as a world line of which a certain segment in temporal extension is activated. This activation may be described by a structure function of the proper time on the world line (with respect to the macroscopic metric pertaining in some chosen primary folium of states). The choice of the world line and of this function are the only data which enter into the description of the detector. Suppose we choose an algebraic element $A$ according to these specifications. Then if $\chi$ is a diffeomorphism which deforms the world line leaving the proper time parametrization unchanged, $\alpha_{\chi} A$ will describe "the same detector" in different motion.

Let us assume now that among the admissible folia there is one containing the vacuum state $\omega_{0}$ of a quantum field theory in flat space so that in the corresponding representation space $\mathfrak{H}_{0}$ the Poincare group is implemented by unitary operators $U(a, \Lambda)$ and the $\omega_{0}^{(n)}$ are the Wightman distributions (vacuum expectation values) of a Minkowski space theory. We have a Hamiltonian $H$ and linear momentum operators $\mathbf{P}$ generating the translations in this folium,

$$
U(a)=e^{i\left(H a^{0}-\mathbf{P a}\right)}, \quad a=\left(a^{0}, \mathbf{a}\right) .
$$

In the remainder of this section we shall be concerned only with this folium and will use Minkowski coordinates $x$ to denote points of $\mathscr{M}$.

To represent a detector at rest at the origin we choose the simplest positive element in the algebra which answers to these specifications. In the notation of ${ }^{7}$ we take

with

$$
C=\phi(f)^{*} \phi(f)
$$

$$
f(x)=\delta^{(3)}(\mathbf{x}) \varphi(t), \quad x=(t, \mathbf{x}) .
$$

If we want to select quanta of energy $E$ we may choose the structure function

$$
\varphi(t)=e^{i E t} e^{-t^{2} / \tau^{2}}
$$

Assume, for simplicity, that the theory describes only one type of particle (mass $m$ ). Denoting $C$ of (2.3) with (2.4), (2.5) inserted by $C(E, \tau)$, we may interpret

$$
d P_{\omega}(E)=\operatorname{const}\left(E^{2}-m^{2}\right)^{-1 / 2} \lim _{\tau \rightarrow \infty} \tau^{-1} \omega(C(E, \tau)) d E \Delta t
$$

as the probability in the state $\omega$ for a particle of energy in the interval $[E, E+d E]$ to hit the detector in the time interval $\Delta t$. The constant depends on the normalization of the field.

We sketch briefly the justification for the interpretation (2.6). If the particle density in the state $\omega$ is low and correlations between particles meeting the world line of the detector at different times may be neglected, then only the matrix elements of $\phi(f)$ between single particle states and the vacuum are relevant and interaction between particles plays no rôle. The matrix element of $\phi(f)$ between a single particle 
state with momentum $p$ and the vacuum is (we omit constant factors)

$$
\int \frac{e^{i(\mathbf{p x}-\varepsilon t)}}{\sqrt{2 \varepsilon}} f(x) d^{4} x=\frac{\tau}{\sqrt{\varepsilon}} e^{-(\varepsilon-E)^{2} \tau^{2} / 4} \equiv A \psi(\mathbf{p}) ; \quad \varepsilon=\left(\mathbf{p}^{2}+m^{2}\right)^{1 / 2},
$$

where $\psi$ is a normalized single particle wave function, i.e. $\int|\psi|^{2} d^{3} \mathbf{p}=1$, and $A^{2}=\tau\left(E^{2}-m^{2}\right)^{1 / 2}$ for large $\tau$. $\psi$ describes a particle with zero angular momentum with an equally large inward and outward directed radial probability current. Its probability of crossing the origin in the future is $\frac{1}{2}$. Thus $A^{-2} \omega(C(E, \tau))$ gives the expected number of particles in the energy range $E \pm \tau^{-1}$ crossing the detector in the time $2 \tau$. This is easily converted into (2.6).

The diffeomorphism $x^{\prime}=\chi x$,

$$
\begin{aligned}
t^{\prime} & =\left(a+x^{(1)}\right) \sinh (t / a), \quad x^{(1)^{\prime}}=\left(a+x^{(1)}\right) \cosh (t / a), \\
x^{(2)^{\prime}} & =x^{(2)} ; \quad x^{(3)^{\prime}}=x^{(3)},
\end{aligned}
$$

sends the world line of the detector at rest at $\mathbf{x}=0$ to the linearly accelerated orbit

$$
x^{(1)^{\prime}}=\left(a^{2}+t^{\prime 2}\right)^{1 / 2} ; \quad x^{(2)^{\prime}}=x^{(3)^{\prime}}=0
$$

such that the proper-time differences for the image points and for the original points are the same. Let us compute the response of the accelerated detector $C^{\prime}$ in the vacuum state $\omega$. We have

$$
\begin{aligned}
C^{\prime}(E, \tau) & =\alpha_{\chi} C(E, \tau)=\phi\left(f^{\prime}\right)^{*} \phi\left(f^{\prime}\right) ; \quad f^{\prime}=f \circ \chi^{-1} . \\
\omega_{0}\left(C^{\prime}(E, \tau)\right) & =\omega_{0}^{(2)}\left(\bar{f}^{\prime} \otimes f^{\prime}\right)=\int d \mu(p) e^{-i p\left(x_{1}^{\prime}-x_{2}^{\prime}\right)} \overline{f^{\prime}}\left(x_{1}^{\prime}\right) f^{\prime}\left(x_{2}^{\prime}\right) d^{4} x_{1}^{\prime} d^{4} x_{2}^{\prime} \\
& =\int d \mu(p) \int d^{4} x_{1} \int d^{4} x_{2} e^{-i p\left(x x_{1}-\chi x_{2}\right)} \overline{f\left(x_{1}\right)} f\left(x_{2}\right)\left|\frac{\partial x_{1}^{\prime}}{\partial x_{1}}\right|\left|\frac{\partial x_{2}^{\prime}}{\partial x_{2}}\right| .
\end{aligned}
$$

Inserting phe definitions (2.4), (2.5) for $f$ and (2.7) for $\chi$, this reduces to

$$
\int d \mu(p) \int d t_{1} d t_{2} e^{-i E\left(t_{1}-t_{2}\right)-i a p^{(0)}\left(\sinh t_{1} / a-\sinh t_{2} / a\right)+i p^{(1)} a\left(\cosh t_{1} / a-\cosh t_{2} / a\right)} \cdot e^{\left(t_{1}^{2}+t_{2}^{2}\right) / \tau^{2}} .
$$

Using the Lorentz invariance of $d \mu(p)$ we get

$$
\omega_{0}\left(C^{\prime}(E)\right)=\lim _{\tau \rightarrow \infty} \tau^{-1} \omega_{0}\left(C^{\prime}(E, \tau)\right)=\int d \rho\left(\kappa^{2}\right) F\left(E, \kappa^{2}\right),
$$

where $d \rho\left(\kappa^{2}\right)$ is the Lehmann-Kallen weight of $\omega_{0}^{(2)}$ and

$$
F\left(E, \kappa^{2}\right)=4 \pi a \int_{\kappa}^{\infty} d \varepsilon\left(\varepsilon^{2}-\kappa^{2}\right)^{1 / 2} \int_{-\infty}^{\infty} d t^{\prime} e^{-i 2 a\left(E t^{\prime}+\varepsilon \sinh t^{\prime}\right)}
$$

Since $\varepsilon$ is always positive we can change the contour of the $t^{\prime}$-integration in the complex plane within the strip Im $\sinh z \leqq 0$ or, putting $z=t-i \alpha(t, \alpha$ real), within $0 \leqq \alpha \leqq \pi$. For the evaluation $\alpha=\pi / 2$ is most convenient, giving

$$
F\left(E, \kappa^{2}\right)=4 \pi a e^{-\pi a E} \int_{\kappa}^{\infty} d \varepsilon\left(\varepsilon^{2}-\kappa^{2}\right)^{1 / 2} \int_{-\infty}^{\infty} d t e^{-i 2 a E t} \cdot e^{-2 a \varepsilon \cosh t} .
$$

For $\kappa=0$ the $\varepsilon$-integration can be performed first, and the result evaluated by the residue theorem. One obtains 


$$
F(E, 0)=4 \pi^{2} E\left(e^{2 \pi a E}-1\right)^{-1} .
$$

For $\kappa \neq 0$ the substitution $\varepsilon=\kappa \cosh u$ and use of the relation

$$
K_{v}(z)=\frac{1}{2} \int d t e^{v t-z \cosh t} ; \quad \operatorname{Re} z>0,
$$

$K_{v}$ denoting the modified Bessel function of the third kind of order $v[6]$, leads to

$$
F\left(E, \kappa^{2}\right)=2 \pi a \kappa^{2} e^{-\pi a E}\left(\left|K_{1-i E a}(a \kappa)\right|^{2}-\left|K_{-i E a}(a \kappa)\right|^{2}\right) .
$$

One sees that the contribution for $\kappa=0$ is the Planck distribution for the HawkingUnruh temperature $k T=(2 \pi a)^{-1}$, while the contribution for $\kappa \neq 0$ is not precisely the corresponding Bose distribution for massive particles in flat space. If, as assumed in (2.6),

$$
d \rho\left(\kappa^{2}\right)=\delta\left(\kappa^{2}-m^{2}\right)+d \rho^{\prime}\left(\kappa^{2}\right),
$$

where $d \rho^{\prime}$ has support starting from $\kappa=2 m$, then for $m \neq 0$ the main contribution to the counting rate comes from $\kappa=m$.

Our purpose in this section was to illustrate in a simple example how the general covariance can be used to compare the response of idealized detectors in different motion. The method can be applied to the more interesting case of circular motion in a synchrotrone [7]. We hope to return elsewhere to a more detailed discussion of such problems. One remark concerning the above mentioned temperature should be added. If one shifts the integration path in (2.10) to $\alpha=\pi$, then one obtains the relation

$$
F\left(E, \kappa^{2}\right)=e^{-2 \pi a E} F\left(-E, \kappa^{2}\right),
$$

which is the KMS-relation and follows directly from the theorems of BisognanoWichmann [8] (see Sect. III of [5] and the literature quoted there). It does, however, not help directly for the computation of counting rates since the commutator $\left[\phi(f)^{*}, \phi(f)\right]$ changes under the diffeomorphism.

\section{The Scaling Limit}

The contents of this section generalize and clarify Sect. II of [5]. The main difference in the point of view is that now $\mathscr{M}$ is not equipped with a given metric field; rather we ultimately want that a primary folium determines a metric field (at least macroscopically). The laws must therefore admit different such folia and a set of germs at a point. Hence the "principle of local definiteness" stressed in [5] must be softened. We can, however, still explore the consequences of the assumption that the theory has a scaling limit in the sense of [5].

Consider a vector field $X$ which vanishes at a point $\bar{P} \in \mathscr{M}$ and is to first order "radial" there, i.e. in a coordinate system (chart $\varphi$ with $\bar{X}=\varphi(\bar{P})$ ) it is of the form

$$
X^{\mu}(x)=x^{\mu}-\bar{x}^{\mu}+O(x-\bar{x})^{2} .
$$

Note that this is an intrinsic property of $X$ since the Kronecker $\delta_{v}^{\mu}$ is invariant. The form of $X^{\mu}(x)$ will differ in different charts only in the quadratic and higher terms in $(x-\bar{x})$. 
The orbits

$$
\frac{d x^{\mu}(\lambda)}{d \lambda}=\lambda^{-1} X^{\mu}(x(\lambda))
$$

will contract an open neighborhood $\mathcal{O}$ of $\bar{P}$ to this point as $\lambda \rightarrow 0$. The solutions of (3.2) with the initial condition $x(1)=x$ define for $\lambda \leqq 1$ a 1-parameter semigroup of local diffeomorphism $\delta_{\lambda}^{X}$ (in multiplicative notation) generated by the vector field,

$$
P \rightarrow \delta_{\lambda}^{X} P \quad \text { corresponding to } \quad x(1) \rightarrow x(\lambda) .
$$

Let $\eta^{X} P$ be the tangent vector of the curve $\delta_{\lambda}^{X} P$ at $\lambda=0 . \eta^{X}: P \rightarrow \eta^{X} P$ is then a diffeomorphism from a neighborhood $\mathcal{O}$ of $\bar{P}$ into the tangent space $\mathscr{T}_{\bar{P}}$. This may be seen by substituting $x^{\mu}(\lambda)=\lambda y^{\mu}(\lambda)$ in (3.2). The mapping $y(1) \rightarrow y(0)$ describes $\eta^{X}$ in terms of the chart $\varphi$. One has

$$
\eta^{X} \delta_{\lambda}^{X} P=\lambda \eta^{X} P
$$

We shall suppress the index $X$ in $\delta_{\lambda}$ and $\eta$ when not essential for distinction. The action of $\delta_{\lambda}$ on the algebra $\mathfrak{A}(\mathcal{O})(\operatorname{see}(2.2))$ will be denoted by $\alpha_{\lambda}$. Similarly $\eta$ defines an isomorphism $\beta$ from $\hat{\mathfrak{U}}(\hat{\mathcal{O}})$, the tensor algebra of test functions in $\mathscr{T}_{\bar{P}}$ with support in $\hat{\mathcal{O}}=\eta \mathcal{O}$, to $\mathfrak{U}(\mathcal{O})$ by

$$
\left(\beta \hat{f}^{(n)}\right)\left(P_{1}, \ldots, P_{n}\right)=\hat{f}^{(n)}\left(\eta P_{1}, \ldots, \eta P_{n}\right)
$$

and, according to (3.4), (2.2) isomorphisms from $\hat{\mathfrak{U}}(\hat{\mathcal{O}})$ to $\hat{\mathfrak{U}}(\lambda \hat{\mathcal{O}})$

$$
\begin{gathered}
\hat{\alpha}_{\lambda}=\beta^{-1} \alpha_{\lambda} \beta \\
\left(\hat{\alpha}_{\lambda} \hat{f}^{(n)}\right)\left(z_{1}, \ldots, z_{n}\right)=\hat{f}^{(n)}\left(\lambda^{-1} z_{1}, \ldots, \lambda^{-1} z_{n}\right) ; \quad z_{i} \in \mathscr{T}_{\bar{P}} .
\end{gathered}
$$

We shall say that a state $\omega$ has a scaling limit at $\bar{P} \in \mathscr{M}$ with respect to the contracting vector field $X$ if there exists a scaling function $N(\lambda)$ (monotone, nonnegative for $\lambda \in(0, \infty])$ such that for all $n \in \mathbb{N}$ and $f^{(n)} \in \mathscr{D}\left(\mathcal{O}^{n}\right)$ the limit of $N(\lambda)^{n} \omega^{(n)}\left(\alpha_{\lambda} f^{(n)}\right)$ for $\lambda \rightarrow 0$ exists and is nonvanishing for some $n, f^{(n) 8}$. Since $\beta$ is a linear positive map we may regard the limit as a state on $\hat{\mathfrak{A}}$ :

$$
\omega_{\bar{P}}^{(n)}\left(\hat{f}^{(n)}\right)=\lim _{\lambda \rightarrow 0} N(\lambda)^{n} \omega^{(n)}\left(\alpha_{\lambda} \beta \hat{f}^{(n)}\right)=\lim _{\lambda \rightarrow 0} N(\lambda)^{n} \omega^{(n)}\left(\beta \hat{\alpha}_{\lambda} \hat{f}^{(n)}\right)
$$

Note that in the last form $\omega_{\bar{P}}$ is defined over the tensor algebra $\hat{\mathfrak{A}}$ of all functions in $\mathscr{D}\left(\mathscr{T}_{\boldsymbol{P}}\right)$ since the support of $\hat{\alpha}_{\lambda} \hat{f}$ will move inside $\hat{\mathcal{O}}$ for sufficiently small $\lambda$ whenever $\hat{f}$ has compact support.

3.1 Theorem. If $\omega$ has a scaling limit at $\bar{P}$ with respect to the contracting vector field $X$, then it has a scaling limit with the same scaling function $N(\lambda)$ for every contracting vector field (in the class (3.1)) and the limit state on the "tangent space algebra" $\hat{\mathfrak{A}}$ is independent of the choice of $X$.

Proof. Suppose $\omega$ has a scaling limit $\omega_{\bar{P}}$ with respect to the vector field $X_{1}$ and

$8 \lambda^{4} N(\lambda) \rightarrow 0$ for $\lambda \rightarrow 0$ if the distributions $\omega^{(n)}$ are more singular than the Lebesgue measure 
scaling function $N(\lambda)$. Let $X_{2}$ be another contracting vector field. Set

$$
\omega_{i}\left(\hat{f}^{(n)} ; \lambda\right)=N(\lambda)^{n} \omega^{(n)}\left(\beta_{i} \hat{\alpha}_{\lambda} \hat{f}^{(n)}\right) ; \quad \beta_{i}=\beta^{X_{i}}, \quad i=1,2 .
$$

Note that $\hat{\alpha}_{\lambda}=\beta_{i}^{-1} \alpha_{\lambda}^{(i)} \beta_{i}$ does not depend on $X_{i}$ due to (3.7). From (3.6) we get

$$
\omega_{2}\left(\hat{f}^{(n)} ; \lambda\right)=\omega_{1}\left(\hat{f}^{(n)} \circ \psi_{\lambda} ; \lambda\right)
$$

with

$$
\begin{aligned}
\psi_{\lambda}(\mathbf{z}) & =\lambda^{-1} \psi(\lambda \mathbf{z}) ; \quad \mathbf{z}=\left(z_{1}, \ldots, z_{n}\right), \\
\psi(\mathbf{z}) & =\eta^{X_{2}}\left(\eta^{X_{1}}\right)^{-1}(\mathbf{z}) .
\end{aligned}
$$

From condition (3.1) we have

$$
\psi(\mathbf{z})=\mathbf{z}+\chi(\mathbf{z}), \text { where } \quad \chi(\lambda \mathbf{z})=O\left(\lambda^{2}\right) .
$$

$\omega_{1}(\lambda)$ is by assumption a pointwise converging sequence of distributions, and therefore uniformly bounded. To prove that $\omega_{2}(\lambda)$ converges and has the same limit as $\omega_{1}(\lambda)$, if suffices therefore to show that for all $\hat{f}^{(n)} \in \mathscr{D}\left(\mathscr{T}_{P}^{n}\right) \hat{f}^{(n)} \circ \psi_{\lambda}$ converges in the topology of $\mathscr{D}\left(\mathscr{T}_{P}^{n}\right)$ to $\hat{f}^{(n)}$, i.e. there is a compact set $\mathscr{K}$ with supp $\hat{f}^{(n)} \circ \psi_{\lambda} \subset \mathscr{K}$ for sufficiently small $\lambda$, and $\hat{f}^{(n)} \circ \psi_{\lambda}$ converges together with all derivatives uniformly on $\mathscr{K}$ to $\hat{f}^{(n)}$.

The support of $\hat{f}^{(n)} \circ \psi_{\lambda}$ is $\psi_{\lambda}^{-1}\left(\operatorname{supp} \hat{f}^{(n)}\right)$. Since

$$
\psi_{\lambda}^{-1}(\mathbf{z})=\lambda^{-1} \psi^{-1}(\lambda \mathbf{z})=\mathbf{z}+\lambda \int_{0}^{\lambda} d \lambda^{\prime}\left(\psi^{-1}\right)^{\prime \prime}\left(\lambda^{\prime} \mathbf{z}\right)(\mathbf{z}, \mathbf{z})
$$

we see that for all $\lambda<\lambda_{0}, \lambda_{0}$ sufficiently small, the support of $f^{(n)} \circ \psi_{\lambda}$ is contained in a compact neighborhood $\mathscr{K}$ of supp $\hat{f}^{(n)}$.

To prove the convergence of the derivatives of $\hat{f}^{(n)} \circ \psi_{\lambda}$ one uses the fact that all derivatives of a composed function $g \circ \varphi, \varphi=\left(\varphi^{1}, \ldots, \varphi^{m}\right), g, \varphi^{i}$ being smooth functions, are finite sums of terms of the form

$$
\left(\partial^{\alpha} g \circ \varphi\right) \partial^{\beta_{1}} \varphi^{j_{1}} \ldots \partial^{\beta_{k}} \varphi^{j_{k}}
$$

with multi-indices $\alpha, \beta_{1}, \ldots, \beta_{k}$, with $j_{1}, \ldots, j_{k} \in\{1, \ldots, m\}$ and $k \in \mathbb{N}$. Therefore it is sufficient to prove that $\partial^{\beta} \psi_{\lambda}$ converges uniformly on $\mathscr{K}$ for all multi-indices $\beta$ to $\partial^{\beta}$ id, where id is the identity mapping.

From the definition of $\psi_{\lambda}$,

$$
\partial^{\beta} \psi_{\lambda}(\mathbf{z})=\lambda^{|\beta|-1} \partial^{\beta} \psi(\lambda \mathbf{z}), \quad|\beta|=\sum_{i=1}^{4 n} \beta_{i} .
$$

Thus for $|\beta| \geqq 2, \partial^{\beta} \psi_{\lambda} \rightarrow 0$, uniformly on $\mathscr{K}$. For the first derivative we obtain

$$
\psi_{\lambda}^{\prime}(\mathbf{z})=\psi^{\prime}(\lambda \mathbf{z}) \rightarrow 1 \text { for } \lambda \rightarrow 0 \text { (uniformly on } \mathscr{K} \text { ) }
$$

and finally

$$
\psi_{\lambda}(\mathbf{z})-(\mathbf{z})=\int_{0}^{1} d t\left(\psi_{\lambda}^{\prime}(t \mathbf{z})-1\right) \mathbf{z} \rightarrow 0
$$

(again uniformly on $\mathscr{K}$ ). This finishes the proof. 
3.2 Theorem. Suppose the state $\omega$ belongs to a primary folium $\mathscr{F}$ and has scaling limit $\omega_{\bar{p}}$. Then, for a dense set of states in $\mathscr{F}$ the scaling limit exists with the same scaling function $N(\lambda)$, and all the limit states coincide with $\omega_{\bar{p}}$.

The proof, demanding a discussion of the relation between $\pi_{\omega}(\mathfrak{X})$ and $\mathfrak{R}_{\omega}$ is deferred to the appendix.

There are a few general properties of scaling limits of distributions.

3.3 Lemma. Let $T \in \mathscr{D}^{\prime}\left(\mathbb{R}^{n}\right)$ and assume that

$$
\lim _{\lambda \rightarrow 0} N(\lambda) T\left(f_{\lambda}\right)=S(f)
$$

exists for all $f \in \mathscr{D}\left(\mathbb{R}^{n}\right)$ and is nonvanishing for some $f$, where $f_{\lambda}(x)=f\left(\lambda^{-1} x\right)$ and $N(\lambda)$ is a monotone positive function. Then $S$ is a tempered distribution and for some $\alpha \in \mathbb{R}$

$$
\lim _{\lambda_{1} \rightarrow 0} \frac{N\left(\lambda_{1}\right)}{N\left(\lambda_{1} \lambda_{2}\right)}=\lambda_{2}^{-\alpha}
$$

b)

$$
S\left(f_{\lambda}\right)=\lambda^{-\alpha} S(f) \text {. }
$$

Proof. $S$ is a distribution due to the sequential completeness of $\mathscr{D}^{\prime}$. Now

$$
S\left(f_{\lambda}\right)=\lim _{\lambda_{1} \rightarrow 0} N\left(\lambda_{1}\right) T\left(f_{\lambda_{1} \lambda}\right)=\lim _{\lambda_{1} \rightarrow 0} \frac{N\left(\lambda_{1}\right)}{N\left(\lambda_{1} \lambda\right)} N\left(\lambda_{1} \lambda\right) T\left(f_{\lambda_{1} \lambda}\right) .
$$

Since $\lim _{\lambda_{1} \rightarrow 0} N\left(\lambda_{1} \lambda\right) T\left(f_{\lambda_{1} \lambda}\right)=S(f)$ exists and does not vanish for some $f$, we have for all $f$

$$
S\left(f_{\lambda}\right)=n(\lambda) S(f) ; \quad n(\lambda)=\lim _{\lambda_{1} \rightarrow 0} \frac{N\left(\lambda_{1}\right)}{N\left(\lambda_{1} \lambda\right)} .
$$

$n(\lambda)$ is continuous because $f_{\lambda}$ depends continuously on $\lambda$ in the sense of the topology of $\mathscr{D}$. It satisfies

$$
n\left(\lambda_{1} \lambda_{2}\right)=n\left(\lambda_{1}\right) n\left(\lambda_{2}\right)
$$

Thus $n(\lambda)$ is a power and we have (3.10),(3.11), i.e. $S$ is a homogeneous distribution of degree $\alpha$, where $\alpha$ is given by (3.10). A homogeneous distribution is always tempered.

We shall now make some assumptions concerning the dependence of the scaling procedure on the contraction point $\bar{P}$.

3.4 Assumption. An admissible primary folium contains states in which the convergence of (3.8) is uniform for $\bar{P}$ ranging through some neighborhood $\mathscr{N}$.

To explain the precise meaning let us use a chart (coordinates $x$ ), identify the tangent spaces at points in the mentioned neighborhood with $\mathbb{R}^{4}$, e.g. by using frames in the tangent space corresponding to the coordinate axes, and let us choose the family of contracting vector fields

$$
X_{\bar{x}}^{\mu}(x)=x^{\mu}-\bar{x}^{\mu}
$$

for $\bar{x}$ ranging through $\mathscr{N}$. Then, defining $\beta_{\bar{x}}$ accordingly (see (3.1) through (3.5)), the assumption means that for fixed $\hat{f}^{(n)} \in \mathscr{D}\left(\mathbb{R}^{4 n}\right)$ there shall be a bound $R_{f}(\lambda)$ with 
$\lim _{\lambda \rightarrow 0} R_{f}(\lambda)=0$ such that

$$
\left|N(\lambda)^{n} \omega^{(n)}\left(\beta_{\bar{x}} \hat{\alpha}_{\lambda} \hat{f}^{(n)}\right)-\omega_{\bar{x}}\left(\hat{f}^{(n)}\right)\right|<R_{f}(\lambda) \text { for all } \bar{x} \in \mathscr{N} .
$$

The proof of Theorem 3.1 shows that this property does not depend on the choice of the chart. We have then

3.5 Theorem. Under Assumption 3.4 the limit states are covariant under dilations and invariant under translations. Specifically, defining

$$
\left(T_{a} \hat{f}^{(n)}\right)\left(z_{1}, \ldots, z_{n}\right)=\hat{f}^{(n)}\left(z_{1}-a, \ldots, z_{n}-a\right),
$$

one has

$$
\begin{aligned}
& \hat{\omega}_{\bar{P}}\left(\hat{\alpha}_{\lambda} \hat{f}^{(n)}\right)=\lambda^{-n \alpha} \hat{\omega}_{\bar{P}}\left(\hat{f}^{(n)}\right), \\
& \hat{\omega}_{\bar{P}}\left(T_{a} \hat{f}^{(n)}\right)=\hat{\omega}_{\bar{P}}\left(f^{(n)}\right) .
\end{aligned}
$$

Proof. The dilation covariance follows trivially from Lemma 3.3. Concerning the translation invariance let us choose a chart $\varphi(\varphi(P)=x)$, identify the tangent spaces with $\mathbb{R}^{4}$ by means of $d \varphi$ and use the family (3.12) of contracting vector fields. Then

$$
\beta_{\bar{x}} \hat{\alpha}_{\lambda} \hat{f}^{(n)}\left(x_{1}, \ldots, x_{n}\right)=\hat{f}^{(n)}\left(\lambda^{-1}\left(x_{1}-\bar{x}\right), \ldots, \lambda^{-1}\left(x_{n}-\bar{x}\right)\right),
$$

and consequently

$$
\beta_{\bar{x}} \hat{\alpha}_{\lambda} T_{a} \hat{f}=\beta_{\bar{x}+\lambda a} \hat{\alpha}_{\lambda} \hat{f}
$$

Put

$$
N(\lambda)^{n} \omega\left(\beta_{\bar{x}+t a} \hat{\alpha}_{\lambda} \hat{f}^{(n)}\right)=F(t, \lambda) .
$$

For fixed $\lambda, F$ is a continuous function of $t$. Choosing an arbitrary $\varepsilon>0$, we can find by Assumption 3.4 a value $\lambda_{1}$ such that $|F(t, \lambda)-F(t, 0)|<\varepsilon / 4$ for $\lambda<\lambda_{1}$ and all $t$ in some fixed interval $|t|<t_{0}$. Due to the continuity in $t$ we can find a $t_{1}<t_{0}$ such that

$$
\left|F\left(t, \lambda_{1}\right)-F\left(0, \lambda_{1}\right)\right|<\frac{\varepsilon}{4} \text { for }|t|<t_{1} .
$$

Then

$$
\begin{aligned}
|F(t, \lambda)-F(0,0)| \leqq & |F(t, \lambda)-F(t, 0)|+F(t, 0)-F\left(t, \lambda_{1}\right) \mid \\
& +\left|F\left(t, \lambda_{1}\right)-F\left(0, \lambda_{1}\right)\right|+\left|F\left(0, \lambda_{1}\right)-F(0,0)\right| .
\end{aligned}
$$

Therefore, for $|t|<t_{1}, \lambda<\lambda_{1}$,

$$
|F(t, \lambda)-F(0,0)|<\varepsilon
$$

By (3.19) the left-hand side of (3.16) is $\lim _{\lambda \rightarrow 0} F(\lambda, \lambda)$. Therefore (3.21) gives the translation invariance (3.16) and also the continuity $\lim _{t \rightarrow 0}\left(\hat{\omega}_{\bar{x}+t a}(\hat{f})-\hat{\omega}_{\bar{x}}(\hat{f})\right)=0$.

The discussion so far has lead to the picture that each admissible primary folium $\mathscr{F}$ determines a section in a fibre bundle $\mathscr{B}$ over $\mathscr{M}$. The fibre over $P \in \mathscr{M}$ is the set of all $\omega_{P}^{\mathscr{F}}$ arising from the different admissible folia by the scaling limit (3.8) at the point 
$P$. A local diffeomorphism $\chi$ of $\mathscr{M}$ induces an action $\alpha_{\chi}^{*}$ on the states (the dual action to (2.2)) and, since the images of all states in one primary folium lie again in one primary folium, it induces an action on the set $\mathscr{F}$ of primary folia which we shall also denote by $\alpha_{\chi}^{*}$. The change of the section $\hat{\omega}^{\mathscr{F}}$ due to the local diffeumorphism $\chi$ is geometrically given:

$$
\hat{\omega}_{P^{\prime}}^{\mathscr{F}^{\prime}}(\hat{f})=\hat{\omega}_{P}^{\mathscr{F}}\left(\hat{f}^{\prime}\right),
$$

where $\mathscr{F}^{\prime}=\alpha_{\chi}^{*} \mathscr{F} ; P^{\prime}=\chi P ; L=L(P, \chi)=(d \chi)_{P}$ is the differential of $\chi$, mapping the tangent space at $P$ on that at $\chi P$ and

$$
\hat{f}^{\prime}\left(z_{1}, \ldots, z_{n}\right)=\hat{f}\left(L z_{1}, \ldots, L z_{n}\right) .
$$

For the subgroup of diffeomorphisms leaving the point $P$ fixed $L$ is a linear transformation of the tangent space at $P$ and (3.23), (3.24) define a corresponding action on the fiber $\hat{\omega}_{p}$. Once we have chosen a frame in the tangent space it determines an action of the group GLR(4) on the fiber.

We shall assume that the fiber consists of a single orbit of this group action. This is the minimal set of states $\hat{\omega}_{P}$ demanded by general covariance, and there is at this stage no reason to consider a larger set. The fibers over different base points must be isomorphic due to (3.23). Therefore, for all points $P$ and all primary folia $\mathscr{F}$ the $\hat{\omega}_{P}^{\mathscr{F}}$ result from a single reference state $\hat{\omega}_{0}$ over the tensor algebra of test functions in $\mathbb{R}^{4}$ by a choice of a frame in the tangent space at $P$ which depends on $\mathscr{F}$. If $\hat{\omega}_{0}$ were not invariant under some subgroup of GLR(4) then each primary folium would define a section in the frame bundle, i.e. it would determine a flat affine connection in $\mathscr{M}$, a teleparallelism. In order to allow curvature $\hat{\omega}_{0}$ must be invariant under some subgroup of GLR(4).

The next question is what this stability subgroup should be. It cannot be the full GLR(4) because there is no translationally invariant 2-point distribution $\hat{\omega}^{(2)}$ which is also invariant under GLR(4). A maximal possible stability group is SLR(4) with either

$$
\hat{\omega}^{(2)}(\hat{f})=c \int \hat{f}\left(z_{1}, z_{2}\right) d^{4} z_{1} d^{4} z_{2}
$$

or

$$
\hat{\omega}^{(2)}(\hat{f})=c \int \hat{f}(z, z) d^{4} z
$$

The first case corresponds to $\alpha=-4$, the second to $\alpha=-2$ in (3.15), (3.10). In both cases each primary folium defines a volume element but not a microscopic metric in the scaling limit. This high symmetry may be an interesting possibility if the philosophy described at the end of Sect. I is adopted.

Another interesting possibility is that the stability subgroup is isomorphic to the Lorentz group. This case corresponds to an essentially classical treatment of gravitation. It is natural then to demand that the tangent space theory at $P$ should satisfy beyond Poincaré invariance also the other standard principles of quantum field theory in the Minkowski space with the metric $g_{P}^{\mathscr{F}}$ defined by $\mathscr{F}$ at $P$, i.e. spacelike commutation and "positive energy." The scaling power is then restricted to values $\alpha \geqq-3$. In the case $\alpha>-3$ the scaling limit of the 2 -point distribution is

$$
\omega^{(2)}\left(z_{1}, z_{2}\right)=\int d \kappa^{2}\left(\kappa^{2}\right)^{\alpha+2} \Delta^{+}\left(z_{1}-z_{2}, \kappa^{2}\right),
$$


where $\Delta^{+}\left(z, \kappa^{2}\right)$ is the positive frequency solution of the Klein-Gordon equation (with respect to the metric $g_{P}^{\mathscr{F}}$ ) with mass $\kappa$; in the case $\alpha=-3$ one obtains

$$
\omega^{(2)}\left(z_{1}, z_{2}\right)=\Delta^{+}\left(z_{1}-z_{2}, 0\right) \equiv D^{+}\left(z_{1}-z_{2}\right) \text {. }
$$

In the latter case the higher truncated functions vanish (see [5]), thus the tangent space theory is the theory of a free massless scalar field.

\section{Reconstruction of the Theory from its Germs}

We address ourselves now to the central question, the compatibility of the strict principle of locality with quantum physics. Is the local information about the laws, as it is contained in the specification of germs, sufficient to determine the laws at large? This is evident in classifical field theory where the only laws are differential equations. In quantum theory we have in addition the commutation relations, and it is not evident how they can propagate.

The basic problem is: Given an open covering $\mathcal{O}=\cup \mathcal{O}_{i}$ and given for each $\mathcal{O}_{i}$ a folium $\mathscr{F}_{i}\left(\mathcal{O}_{i}\right)$, i.e. the equivalence class ${ }^{9}$ of a representation $\pi_{i}$ of $\mathfrak{A}\left(\mathcal{O}_{i}\right)$,

1) can there exist different folia $\mathscr{F}(\mathcal{O})$ whose restrictions to the $\mathcal{O}_{i}$ are the given ones?

2) What are the compatibility conditions for the set $\left\{\mathscr{F}_{i}\left(\mathcal{O}_{i}\right)\right\}$ so that an extension $\mathscr{F}(\mathcal{O})$ exists?

A full analysis of these questions is beyond the scope of the present paper. We shall show, however, that in a somewhat different sense the theory can be constructed from strictly local information. The input then is a "tame state" $\omega_{i}$ for each region $\mathcal{O}_{i}$ of the covering; compatibility means that the restrictions of these partial states to the overlap regions $\mathcal{O}_{i} \cap \mathcal{O}_{j}$ must coincide. The "extended theory" to the covered region $\mathcal{O}$ means an additive net of von Neumann algebras in $\mathcal{O}$, coordinatized in the small by affiliated Borchers algebras. It is uniquely determined by the $\left\{\omega_{i}\right\}$. In general the Borchers algebra of the large region will no longer be affiliated with the von Neumann algebra on $\mathcal{O}$. It is not clear whether this discrepancy is a weakness or a strength of this construction. If one wants to retain the correspondence between von Neumann algebras and Borchers algebras in the large then stronger compatibility conditions must be imposed. They should be conditions for local equilibrium of the set $\left\{\omega_{i}\right\}$ and may imply that the construction of the theory can be done-as suggested by the questions above - using as local information only the set of partial folia $\left\{\mathscr{F}_{i}\right\}$.

We recall that in ordinary quantum field theory the positivity of the energy leads to analyticity of Wightman functions and that this, in turn, as shown by Reeh and Schlieder [9], implies that the subset of state vectors obtained by applying the algebra of any open region to the vacuum is already dense in the Hilbert space of all state vectors. In short, the net of algebras $\left\{\pi_{0}(\mathfrak{A}(\mathcal{O}))\right\}$ has a common cyclic vector for all open regions. In fact it has many such vectors since any state with finite energy

9 We should be speaking of "quasiequivalence classes" but since we anticipate dealing with type III factors quasiequivalence and unitary equivalence coincide 
has this property. Let us call a state $\omega$ over $\mathfrak{A}(\mathcal{O})$ "fully correlated within $\mathcal{O}$ " if in the GNS-representation the set of vectors $\pi_{\omega}(\mathfrak{U}(\widetilde{\mathcal{O}})) \Omega$ is dense in $\mathfrak{H}_{\omega}$ for any open subregion $\tilde{\mathcal{O}} \subset \mathcal{O}$. One has then

4.1 Theorem ${ }^{10}$. Let $\mathcal{O}$ be open and connected, $\mathcal{O}=\bigcup_{i \in J} \mathcal{O}_{i}$ an open covering and $\omega$ a state on $\mathfrak{U}(\mathcal{O})$ which is fully correlated within $\mathcal{O}$. Then there exists no other state on $\mathfrak{U}(\mathcal{O})$ whose restrictions to the subalgebras $\mathfrak{A}\left(\mathcal{O}_{i}\right)$ coincide with those of $\omega$.

Proof. Suppose $\omega^{\prime}$ is another state on $\mathfrak{A}(\mathcal{O})$, not assumed to be fully correlated on $\mathcal{O}$ but satisfying

$$
\left(\omega^{\prime}-\omega\right) \uparrow \mathfrak{U}\left(\mathcal{O}_{i}\right)=0 ; \quad i \in J
$$

Let $(\mathfrak{H}, \pi, \Omega)$ respectively $\left(\mathfrak{G}^{\prime}, \pi^{\prime}, \Omega^{\prime}\right)$ be the GNS-representations induced by $\omega$ respectively $\omega^{\prime}$. Due to (4.1) we may put for each $i \in J$,

$$
V_{i} \pi(A) \Omega=\pi^{\prime}(A) \Omega^{\prime} ; \quad A \in \mathfrak{U}\left(\mathcal{O}_{i}\right)
$$

and, since $\pi\left(\mathfrak{U}\left(\mathcal{O}_{i}\right)\right) \Omega$ is dense in $\mathfrak{H}$, by assumption $V_{i}$ is an isometric map from $\mathfrak{H}$ into $\mathfrak{Y}^{\prime}$ with

$$
V_{i} \pi(A)=\pi^{\prime}(A) V_{i} ; \quad A \in \mathfrak{U}\left(\mathcal{O}_{i}\right)
$$

If $\mathcal{O}_{i} \cap \mathcal{O}_{j} \neq \phi$, we have

$$
V_{i} \pi(A) \Omega=V_{j} \pi(A) \Omega ; \quad A \in \mathfrak{U}\left(\mathcal{O}_{i} \cap \mathcal{O}_{j}\right) .
$$

But by assumption $\pi\left(\mathfrak{U}\left(\mathcal{O}_{i} \cap \mathcal{O}_{j}\right)\right) \Omega$ is again dense in $\mathfrak{H}$. Thus $V_{i}=V_{j}$. This leads to the equality of all $V_{i}$ :

$$
V_{i}=V ; \quad i \in J,
$$

for, if (4.4) would hold for a subset $J_{0} \subset J$ we could obtain $\mathcal{O}$ as the union of the two open sets

$$
\hat{\mathcal{O}}=\bigcup_{i \in J_{0}} \mathcal{O}_{i} ; \quad \hat{\mathcal{O}}=\bigcup_{i \in J_{0}^{c}} \mathcal{O}_{i}
$$

which are disjoined. As $\mathcal{O}$ was assumed to be connected $\hat{\mathcal{O}}=\phi$. Now we get for a product of elements from different regions

$$
\begin{aligned}
\omega^{\prime}\left(\prod_{1}^{n} A_{i}\right) & =\left(\Omega^{\prime}, \prod_{1}^{n} \pi^{\prime}\left(A_{i}\right) \Omega^{\prime}\right)=\left(V \Omega, \prod_{1}^{n-1} \pi^{\prime}\left(A_{i}\right) V \pi\left(A_{n}\right) \Omega\right) \\
& =\left(V \Omega, V \prod_{1}^{n} \pi\left(A_{i}\right) \Omega\right)=\omega\left(\prod_{1}^{n} A_{i}\right),
\end{aligned}
$$

where (4.3), (4.4) are used to move $V$ successively to the left on the right-hand side of the equation. Thus $\omega^{\prime}$ coincides with $\omega$ on the algebra generated by the $\mathfrak{A}\left(\mathcal{O}_{i}\right)$ which 
is $\mathfrak{A}(\mathcal{O})$ in the case of the Borchers algebra or, more generally, whenever $\mathfrak{A}$ is an additive net.

We turn now to the construction of a state over $\mathfrak{A}(\mathcal{O})$ from the partial states $\omega_{i}$ of a covering. Let $\mathcal{O}_{1} \cap \mathcal{O}_{2} \neq \phi$ and $\omega_{i}(i=1,2)$ states over $\mathfrak{A}\left(\mathcal{O}_{i}\right)$ respectively fully correlated within their regions of definition and compatible in the intersection region:

$$
\omega_{1}(A)=\omega_{2}(A) ; \quad A \in \mathfrak{A}\left(\mathcal{O}_{1} \cap \mathcal{O}_{2}\right) .
$$

Denote the GNS-representations of $\mathfrak{A}\left(\mathcal{O}_{i}\right)$ induced by $\omega_{i}$ by $\left(\mathfrak{H}_{i}, \Omega_{i}, \pi_{i}\right)$. Then (4.5) together with the cyclicity of $\Omega_{i}$ with respect to $\pi_{i}\left(\mathfrak{H}\left(\mathcal{O}_{1} \cap \mathcal{O}_{2}\right)\right)$ implies that we have a unitary map $V_{12}$ from $\mathfrak{H}_{2}$ onto $\mathfrak{H}_{1}$ uniquely determined by

$$
\begin{gathered}
V_{12} \Omega_{2}=\Omega_{1}, \\
V_{12} \pi_{2}(A) V_{12}^{-1}=\pi_{1}(A) ; \quad A \in \mathfrak{A}\left(\mathcal{O}_{1} \cap \mathcal{O}_{2}\right) .
\end{gathered}
$$

If we have three regions with $\mathcal{O}_{1} \cap \mathcal{O}_{2} \cap \mathcal{O}_{3} \neq \phi$ and

$$
\omega_{i}(A)=\omega_{j}(A) ; \quad A \in \mathfrak{H}\left(\mathcal{O}_{i} \cap \mathcal{O}_{j}\right),
$$

then the unitaries satisfy

$$
V_{12} V_{23}=V_{13} \text {. }
$$

4.2 Theorem ${ }^{11}$. Let $\mathcal{O}=\bigcup_{i \in J} \mathcal{O}_{i}$ be a covering of a path connected, simply connected topological space $\mathcal{O}$ by path connected open sets, let $\mathfrak{H}_{i}, i \in J$ be a family of Hilbert spaces, and let for each pair $i, j \in J$ with $\mathcal{O}_{i} \cap \mathcal{O}_{j} \neq \phi V_{i j}$ be a unitary operator from $\mathfrak{H}_{j}$ onto $\mathfrak{H}_{i}$ such that

$$
V_{i j} V_{j k}=V_{i k} \quad \text { if } \quad \mathcal{O}_{i} \cap \mathcal{O}_{j} \cap \mathcal{O}_{k} \neq \phi .
$$

Then there exists a Hilbert space $\mathfrak{S}$ and a family of unitary operators $W_{i}: \mathfrak{H}_{i} \rightarrow \mathfrak{G}, i \in J$ such that

$$
V_{i j}=W_{i}^{-1} W_{j} .
$$

Proof. Fix $i_{0} \in J$ and $x_{0} \in \mathcal{O}_{i_{0}}$. Let $j \in J$ and $y \in \mathcal{O}_{j}$. Since $\mathcal{O}$ is path connected there is a path $\gamma$ from $x_{0}$ to $y$, i.e. a continuous mapping $\gamma$ from the unit interval $[0,1]$ into $\mathcal{O}$ with $\gamma(0)=x_{0}$ and $\gamma(1)=y$. For each $k \in J \gamma^{-1}\left(\mathcal{O}_{k}\right)$ is an open subset of the unit interval. Its connected components are intersections of open intervals with the unit interval.

Let $C_{k}$ denote the set of connected components of $\gamma^{-1}\left(\mathscr{O}_{k}\right)$, and let $\mathscr{C}=\mathscr{C}(\gamma)$ be the disjoint union of the sets $C_{k}, k \in J . \mathscr{C}$ is a covering of $[0,1]$ by open intervals. For $I \in C_{k}$ we set $k(I)=k$. We have $\mathcal{O}_{k(I)} \cap \mathcal{O}_{k\left(I^{\prime}\right)} \neq \phi$ if $I \cap I^{\prime} \neq \phi$ and $\mathcal{O}_{k(I)} \cap \mathcal{O}_{k\left(I^{\prime}\right)} \cap \mathcal{O}_{k\left(I^{\prime \prime}\right)} \neq \phi$ if $I \cap I^{\prime} \cap I^{\prime \prime} \neq \phi$.

Since the unit interval is compact, it can be covered by finitely many intervals

11 This theorem was stated by J. E. Roberts [11] and proved by him in the context of non-Abelian cohomology 
$I_{0}, \ldots, I_{n} \in \mathscr{C}$. We can choose them such that $0 \in I_{0} \in C_{i_{0}}, 1 \in I_{n} \in C_{j}$ and $I_{l} \cap I_{l+1} \neq \phi$, $l=0, \ldots, n-1$. Using such a sequence of intervals we define $W_{j}$ by

$$
W_{j}=V_{k\left(I_{0}\right) k\left(I_{1}\right)} \cdots V_{k\left(I_{n-1}\right) k\left(I_{n}\right)} \text {. }
$$

We have to show that $W_{j}$ does neither depend on the choice of the intervals $I_{0}, \ldots, I_{n}$ nor on the path $\gamma$ from $x_{0}$ to $y$ nor on the choice of $y \in \mathcal{O}_{j}$.

Provided this can be achieved the proof is completed as follows. Given $i \in J$ with $\mathcal{O}_{i} \cap \mathcal{O}_{j} \neq \phi$, we take $y \in \mathcal{O}_{i} \cap \mathcal{O}_{j}$ and a path $\gamma$ from $x$ to $y$. We can select the sequence of intervals such that $I_{n-1} \in C_{i}$. Then $V_{k\left(I_{n-1}\right) k\left(I_{n}\right)}=V_{i j}$ and

$$
W_{i} V_{i j}=W_{j} \text {. }
$$

The independence of $W_{j}$ from the choice of the intervals $I_{0}, \ldots, I_{n}$ is equivalent to the statement that

$$
V_{D} \equiv V_{k\left(D_{1}\right) k\left(D_{2}\right)} \cdots V_{k\left(D_{n-1}\right) k\left(D_{n}\right)} V_{k\left(D_{n}\right) k\left(D_{1}\right)}=1
$$

for any sequence of intervals $D_{1}, \ldots, D_{m} \in \mathscr{C}$ with $D_{l} \cap D_{l+1} \neq \phi, l=1, \ldots, m-1$ and $D_{m} \cap D_{1} \neq \phi$. For $m=2$, (4.14) follows directly from the cocycle condition (4.10). For general $m$ we proceed by induction and assume that (4.14) holds for any such sequence of $(m-1)$ intervals. Let $D_{\ell}=\left(a_{\ell}, b_{\ell}\right), a_{\ell}<b_{\ell}, \ell=1, \ldots, m$ and let $a_{r}=\min a_{\ell}$. Then either $D_{r+1} \subset D_{r}, D_{r-1} \subset D_{r}$ or $b_{r} \in D_{r-1} \cap D_{r+1}$ (we set $D_{\ell}=D_{\ell \text { modm }}$ for $\ell \in \mathbb{Z}$ ). In all cases three subsequent intervals have a nonempty intersection,

$$
D_{s-1} \cap D_{s} \cap D_{s+1} \neq \phi, \quad s=r-1, \quad r \quad \text { or } r+1,
$$

thus

$$
V_{k\left(D_{s-1}\right) k\left(D_{s}\right)} V_{k\left(D_{s}\right) k\left(D_{s+1}\right)}=V_{k\left(D_{s-1}\right) k\left(D_{s+1}\right)}
$$

and

$$
V_{D}=S V_{D^{\prime}} S^{-1}
$$

with $D_{\ell}^{\prime}=D_{\ell}, \ell=1, \ldots, s-1, D_{\ell}^{\prime}=D_{\ell+1}, \ell=s, \ldots, m-1$ and $S=1$ for $s \neq 1, S=$ $V_{k\left(D_{1}\right) k\left(D_{2}\right)}$ for $s=1$. By the induction hypothesis $V_{D^{\prime}}=1$, hence $V_{D}=1$.

In the next step we show the invariance of $W_{j}$ under continuous deformations of the path $\gamma$. Since $\mathcal{O}$ is simply connected this implies the independence of $W_{j}$ from $\gamma$. Let $\alpha$ be a continuous mapping from the unit square $[0,1] \times[0,1]$ into $\mathcal{O}$ such that

$$
\begin{aligned}
& \alpha(0, t)=\gamma(t), \quad t \in[0,1], \\
& \alpha(s, 0)=x_{0}, \quad \alpha(s, 1)=y, \quad s \in[0,1] .
\end{aligned}
$$

Let $\gamma_{s}(t)=\alpha(s, t), s, t \in[0,1]$, and let $W_{j}(s)$ be the unitary operator $W_{j}$ associated to $\gamma_{s}$. Let $I_{\ell}=\left(a_{\ell}, b_{\ell}\right) \cap[0,1], a_{\ell}<b_{\ell}, \ell=0, \ldots, n$ be a sequence of intervals in $\mathscr{C}$ which fulfils the conditions mentioned before relation (4.12). Then for $\varepsilon$ sufficiently small, there are intervals $I_{\ell}(s)=\left(a_{\ell}(s), b_{\ell}(s)\right)$ with $b_{\ell}(s)>a_{\ell+1}(s), \ell=0, \ldots, n-1$, $0 \in I_{0}(s), 1 \in I_{n}(s), 0 \leqq s<\varepsilon$ which are connected components of $\gamma_{s}^{-1}\left(\mathcal{O}_{k\left(I_{\ell}\right)}\right)$. Inserting this sequence in (4.12) we get

$$
W_{j}(s)=W_{j} \text { for } 0 \leqq s<\varepsilon,
$$

hence $W_{j}$ is constant under continuous deformations of $\gamma$. 
It remains to show that $W_{j}$ does not depend on the choice of $y \in \mathcal{O}_{j}$. Let $\tilde{y}$ be another point in $\mathcal{O}_{j}$. Since $\mathcal{O}_{j}$ is path connected, there is a path $\tilde{\gamma}$ in $\mathcal{O}_{j}$ from $y$ to $\tilde{y}$. Let $\gamma^{\prime}=\tilde{\gamma} \gamma$ be the composed path. Then the connected component of the intersection of the path $\gamma^{\prime}$ with $\mathcal{O}_{j}$ with contains $y$ also contains $\tilde{y}$, thus the intervals $I_{\ell}^{\prime} \in \mathscr{C}\left(\gamma^{\prime}\right)$ which enter in (4.12) can be chosen such that $k\left(I_{\ell}^{\prime}\right)=k\left(I_{\ell}\right)$, hence $W_{j}$ does not change. This finishes the proof.

We conclude: given a covering $\mathcal{O}=\cup \mathcal{O}_{i}$ as specified in Theorem 4.2 and given a fully correlated partial state $\omega_{i}$ on each $\mathcal{O}_{i}$ such that the system $\left\{\omega_{i}\right\}$ is compatible in the intersection regions in the sense of $(4.8)$, then there is a Hilbert space $\mathfrak{H}$ with a vector $\Omega$ and a system of operator algebras in $\mathfrak{H}$

$$
\tilde{\pi}_{i}\left(\mathfrak{U}\left(\mathcal{O}_{i}\right)\right)=W_{i} \pi_{\omega_{\imath}}\left(\mathfrak{U}\left(\mathcal{O}_{i}\right)\right) W_{i}^{-1}
$$

such that $\tilde{\pi}_{i}$ is a representation of $\mathfrak{U}\left(\mathcal{O}_{i}\right)$, the system $\left\{\tilde{\pi}_{i}\right\}$ is compatible in the sense

$$
\tilde{\pi}_{i}(A)=\tilde{\pi}_{j}(A) ; \quad A \in \mathfrak{A}\left(\mathcal{O}_{i} \cap \mathcal{O}_{j}\right)
$$

and

$$
\left(\Omega, \tilde{\pi}_{i}(A) \Omega\right)=\omega_{i}(A) ; \quad A \in \mathfrak{U}\left(\mathcal{O}_{i}\right), i \in J
$$

Furthermore $\Omega$ is cyclic for $\tilde{\pi}_{i}(\mathfrak{U}(\tilde{\mathcal{O}}))$ whenever $i \in J$ and $\tilde{\mathcal{O}} \subset \mathcal{O}_{i}$ is open. Conversely, the structure $\left(\mathfrak{H}, \Omega, \tilde{\pi}_{i}\right)$ is uniquely determined up to unitary equivalence by the requirements that $\tilde{\pi}_{i}$ are representations of $\mathfrak{U}\left(\mathcal{O}_{i}\right)$ satisfying (4.16) and that $\Omega$ satisfies (4.17) and has the stated cyclicity properties ${ }^{12}$.

Does the system $\tilde{\pi}_{i}$ define a representation of $\mathfrak{U}(\mathcal{O})$ ? There are two possible obstacles. First, with our choice of $\mathfrak{U}$ (the Borchers algebra) the operator $\tilde{\pi}_{i}(A)$ will be unbounded in general, and we need a common, dense, invariant domain to be able to multiply operators coming from different $i \in J$. Only then can we generate an operator algebra

$$
\tilde{\mathfrak{A}}=\bigvee_{i \in J} \tilde{\pi}_{i}\left(\mathfrak{A}\left(\mathcal{O}_{i}\right)\right)
$$

Secondly, if $\mathfrak{\mathscr { U }}$ exists we have to verify that it is a representation, i.e. that no relations within $\mathfrak{U}(\mathcal{O})$ are violated in $\tilde{\mathfrak{U}}$. This will certainly be true if $\mathfrak{U}(\mathcal{O})$ is free over $\left\{\mathfrak{U}\left(\mathcal{O}_{i}\right)\right\}$ in the sense of the following definition.

4.3 Definition. Let $\mathfrak{U}_{i}, i \in J$ be a family of subalgebras of $\mathfrak{A}$. $\mathfrak{A}$ is called free over $\left\{\mathfrak{U}_{i}, i \in J\right\}$ if the following holds: For any algebra $\widetilde{\mathfrak{U}}$ for which there exist morphisms $\gamma_{i}: \mathfrak{I}_{i} \rightarrow \tilde{\mathfrak{A}}$ satisfying the compatibility condition

$$
\gamma_{i} \uparrow \mathfrak{U}_{i} \cap \mathfrak{A}_{j}=\gamma_{j} \uparrow \mathfrak{U}_{i} \cap \mathfrak{U}_{j},
$$

there is a morphism $\gamma: \mathfrak{A} \rightarrow \tilde{\mathfrak{U}}$ with $\gamma \uparrow \mathfrak{U}_{i}=\gamma_{i}$. One checks

12 For the purpose of studying the significance of local gauge invariance in the algebraic frame of quantum field theory in Minkowski space, J. E. Roberts [11] considered such a system of representations $\tilde{\pi}_{i}$ of a given net of von Neumann algebras. He called it a "local representation." The extension problem encountered there is not present in our simple model but may be very relevant in more realistic theories 
4.4 Theorem. Let $\mathcal{O}=\cup \mathcal{O}_{i}$ be an open covering of a differentiable manifold $\mathcal{O}$. Then the Borchers algebra $\mathfrak{U}(\mathcal{O})$ is free over $\left\{\mathfrak{U}\left(\mathcal{O}_{i}\right), i \in J\right\}$.

Proof. Let $\gamma_{i}: \mathfrak{A}\left(\mathcal{O}_{i}\right) \rightarrow \widetilde{\mathfrak{A}}, i \in J$ be a family of morphisms into an algebra $\tilde{\mathfrak{A}}$ with $\left(\gamma_{i}-\gamma_{j}\right)(A)=0$ for $A \in \mathfrak{U}\left(\mathcal{O}_{i} \cap \mathcal{O}_{j}\right)$. Let $f \in \mathscr{D}(\mathcal{O})$ and $\mathscr{K} \subset \mathcal{O}$ compact with supp $f \subset \mathscr{K}$. There is a finite $\mathscr{C}^{\infty}$-decomposition of the identity on $\mathscr{K}$ subordinate to $\left\{\mathcal{O}_{i}, i \in J\right\}$, i.e. a finite family $\left\{\varphi_{i} \in \mathscr{D}\left(\mathcal{O}_{i}\right)\right\}$ with $\sum \varphi_{i}(x)=1$ for $x \in \mathscr{K}$. Since $f \varphi_{i} \in \mathscr{D}\left(\mathcal{O}_{i}\right) \subset \mathfrak{A}\left(\mathcal{O}_{i}\right)$ we can set

$$
\gamma(f)=\sum \gamma_{i}\left(f \varphi_{i}\right)
$$

If $\left\{\psi_{j} \in \mathscr{D}\left(\mathcal{O}_{j}\right)\right\}$ is another finite $\mathscr{C}^{\infty}$-decomposition of the identity on $\mathscr{K}$, we have $f \varphi_{i} \psi_{j} \in \mathscr{D}\left(\mathcal{O}_{i} \cap \mathcal{O}_{j}\right)$, and therefore

$$
\sum_{i} \gamma_{i}\left(f \varphi_{i}\right)=\sum_{i, j} \gamma_{i}\left(f \varphi_{i} \psi_{j}\right)=\sum_{i, j} \gamma_{j}\left(f \varphi_{i} \psi_{j}\right)=\sum_{j} \gamma_{j}\left(f \psi_{j}\right)
$$

hence $\gamma(f)$ does not depend on the choice of the family $\left(\varphi_{i}\right)$. Since for any compact set $\mathscr{K}^{\prime} \supset \mathscr{K}$ we can enlarge the family $\left(\varphi_{i}\right)$ to a finite decomposition of the identity on $\mathscr{K}^{\prime}$ by adding functions vanishing on $\mathscr{K}, \gamma(f)$ also does not depend on the choice of $\mathscr{K}$. Hence $\gamma$ is a well defined linear map from $\mathscr{D}(\mathcal{O})$ into $\tilde{\mathfrak{A}}$. Moreover, if $f \in \mathscr{D}\left(\mathcal{O}_{j}\right), f \varphi_{i} \in \mathscr{D}\left(\mathcal{O}_{i} \cap \mathcal{O}_{j}\right)$, hence

$$
\gamma(f)=\sum_{i} \gamma_{i}\left(f \varphi_{i}\right)=\sum_{i} \gamma_{j}\left(f \varphi_{i}\right)=\gamma_{j}(f)
$$

Since $\mathfrak{A}(\mathcal{O})$ is the tensor algebra over $\mathscr{D}(\mathcal{O}), \gamma$ has a unique extension to a morphism of $\mathfrak{U}(\mathcal{O})$ into $\tilde{\mathfrak{U}}$, and $\gamma(A)=\gamma_{i}(A)$ for $A \in \mathfrak{U}\left(\mathcal{O}_{i}\right)$.

Thus $\tilde{\mathfrak{A}}$ in (4.18), if it exists, i.e. if the multiplication is not obstructed by domain problems, is a representation $\pi$ of $\mathfrak{A}(\mathcal{O})$. The state

$$
\omega(A)=(\Omega, \pi(A \Omega) ; \quad A \in \mathfrak{U}(\mathcal{O})
$$

extends the given $\omega_{i}$ and is fully correlated on $\mathcal{O}$. Due to Theorem $4.1 \omega$ is the only possible extension of $\left\{\omega_{i}\right\}$ and $\pi$ the only possible extension of $\left\{\pi_{\omega_{i}}\right\}$ to $\mathcal{O}$. The conditions used are that the covering is as specified at the beginning of Theorem 4.2, that each $\omega_{i}$ is fully correlated within its region of definition, that the natural compatibility condition (4.8) for the intersection regions is respected and last, but not least, that the domains of the $\tilde{\pi}_{i}$ fit together.

To assess the physical significance of the last probelm we must remember that we would prefer to obtain an extension of the set of partial folia $\left\{\mathscr{F}_{i}\right\}$ (which define "the laws" in the small) and not one of individual states. If each $\mathscr{F}_{i}$ contains a fully correlated state $\omega_{i}$, then $\mathscr{F}_{i}$ is determined by $\omega_{i}$ but not vice versa. Therefore, if we hope that the specification of $\left\{\mathscr{F}_{i}\right\}$ determines a unique extension $\mathscr{F}$ to the covered region $\mathcal{O}$, we need stronger restrictions on the choice of the $\omega_{i}$ and stronger compatibility requirements in the construction described above. Concerning the restrictions for the choice of each partial state $\omega_{i}$ a guide line is the requirement that $\omega_{i}$ should generate within $\mathcal{O}_{i}$ a well defined additive net of von Neumann algebras $\mathfrak{R}_{\omega_{i}}(\tilde{\mathcal{O}}), \tilde{\mathcal{O}} \subset \mathcal{O}_{i}$, where $\mathfrak{R}_{\omega_{i}}(\widetilde{\mathcal{O}})$ is obtained from $\omega_{i} \uparrow \tilde{\mathcal{O}}$ by the construction described in the appendix. Let us call a state $\omega_{i}$ on $\mathfrak{U}\left(\mathcal{O}_{i}\right)$ "tame" if it generates an additive net $\mathfrak{R}_{i}$ 
within $\mathcal{O}_{i}$. Tame states must be fully correlated and satisfy additional conditions ${ }^{13}$. Concerning the compatibility of the set $\left\{\omega_{i}\right\}$ we note that if $\omega_{1}$ is given and $\mathcal{O}_{1} \cap \mathcal{O}_{2} \neq \phi$, then we can change $\omega_{2}$ by a unitary from $\mathfrak{R}_{\omega_{2}}^{\prime}\left(\mathcal{O}_{1} \cap \mathcal{O}_{2}\right)$ without changing the folium $\mathscr{F}_{2}$ or the relation (4.8). This freedom of the choice of $\omega_{2}$ within $\mathscr{F}_{2}$ is reduced if one requires that the domains of $\tilde{\pi}_{1}$ and $\tilde{\pi}_{2}$ shall fit together. This requirement will also demand a stronger compatibility condition between $\mathscr{F}_{1}$ and $\mathscr{F}_{2}$ than the naive one that they agree on $\mathcal{O}_{1} \cap \mathcal{O}_{2}$. It may lead to a unique determination of the extended folium $\mathscr{F}$ to $\mathcal{O}$ from $\left\{\mathscr{F}_{1}, \mathscr{F}_{2}\right\}$ alone. As mentioned at the beginning of this section we would interpret such conditions as a demand of local equilibrium. We shall not pursue this line further here.

Irrespective of these last speculations the results of this section show that in a weaker sense an extension of the theory to $\mathcal{O}$ from local information on a covering $\left\{\mathcal{O}_{i}\right\}$ is possible and unique. Namely, specifying a set $\left\{\omega_{i}\right\}$ of tame states on the covering respecting (4.8) we obtain first additive nets $\Re_{i}$ of von Neumann algebras in $\mathfrak{S}_{\omega_{i}}$ with an identification of elements,

$$
X \in \mathfrak{R}_{i}\left(\mathcal{O}_{i} \cap \mathcal{O}_{j}\right) \rightarrow \gamma_{j i} X \in \mathfrak{R}_{j}\left(\mathcal{O}_{i} \cap \mathcal{O}_{j}\right),
$$

given by the common affiliation of $\mathfrak{A}\left(\mathcal{O}_{i}, \cap \mathcal{O}_{j}\right)$ with these two von Neumann algebras. We can then regard $\omega_{i}$ as a state on $\mathfrak{R}_{i}\left(\mathcal{O}_{i}\right)$ and obtain a system of von Neumann algebras $\widetilde{R}_{i}$ within the bounded operators of a single Hilbert space $\mathfrak{H}$ and $\Omega \in \mathfrak{H}$, replacing (4.15)-(4.17) by

$$
\begin{aligned}
\tilde{\pi}_{i}(X) & =W_{i} X W_{i}^{-1}: \quad X \in \mathfrak{R}_{i}\left(\mathcal{O}_{i}\right), \\
\tilde{\pi}_{i}(X) & =\tilde{\pi}_{j} \gamma_{j i}(X) ; \quad X \in \mathfrak{R}_{i}\left(\mathcal{O}_{i} \cap \mathcal{O}_{j}\right), \\
\left(\Omega, \tilde{\pi}_{i}(X) \Omega\right) & =\omega_{i}(X) ; \quad X \in \mathfrak{R}_{i}\left(\mathcal{O}_{i}\right) .
\end{aligned}
$$

Then

$$
\tilde{\mathfrak{R}}(\mathcal{O})=\bigvee_{i \in J} \tilde{\pi}_{i}\left(\mathfrak{R}_{i}\right)
$$

is well defined and gives an additive net of von Neumann algebras over $\mathcal{O}$. The vector $\Omega$ gives a state on $\widetilde{R}(\mathcal{O})$ which is fully correlated within $\mathcal{O}$ and this pair $(\widetilde{R}, \omega)$ is the only possible extension of the system $\left\{\Re_{i}, \omega_{i}\right\}$ up to unitary equivalence. In general $\mathfrak{H}(\mathcal{O})$ will no longer be affiliated with $\widetilde{\mathfrak{R}}(\mathcal{O})$, but this may not be relevant. The only rôle which the Borchers algebra plays now is to define the identification map $\gamma_{j i}$ of the elements of $\mathfrak{R}_{i}\left(\mathcal{O}_{i} \cap \mathcal{O}_{j}\right)$ and $\mathfrak{R}_{j}\left(\mathcal{O}_{i} \cap \mathcal{O}_{j}\right)$. However, the identification of elements in the intersection region is already uniquely determined by the geometric interpretation given by the net structure in $\mathscr{O}_{i} \cap \mathcal{O}_{j}$ unless $\mathfrak{R}_{i}$ possesses automorphisms transforming every subalgebra $\mathfrak{R}_{i}(\hat{\mathcal{O}}), \hat{\mathcal{O}} \subset \mathcal{O}_{i}$ into itself, i.e. in physical terms unless there exist internal symmetries. In that case, however, we face the problem indicated in $^{12}$ which we want to leave aside here. Thus one might drop the Borchers algebra and base the theory directly on local nets of von Neumann algebras in the small.

The problems discussed find a natural expression in the framework of sheaf 
theory. As an illustration let us consider the presheaf $\mathcal{O} \rightarrow \mathscr{S}_{c}(\mathcal{O})$, the set of fully correlated states within $\mathcal{O}$. The strictly local part of the information in $\mathscr{S}_{c}$ can be described by the associated sheaf $\tilde{\mathscr{S}}_{c}=\cup \mathscr{S}_{c}(x)$, where $\mathscr{S}_{c}(x)$ is the set of germs of states in $\mathscr{S}_{c}$ at the point $x$. The topology in $\widetilde{\mathscr{S}}_{c}$ is given in terms of a basis of the system of open sets,

$$
\mathscr{B}_{\omega, \mathcal{O}}=\left\{g_{y} \mid g_{y} \quad \text { germ of } \omega \text { at } y, \quad y \in \mathcal{O}\right\},
$$

where $\mathcal{O}$ is open and $\omega \in \mathscr{S}_{c}(\mathcal{O})$.

Now let $s: x \rightarrow s_{x} \in \mathscr{S}_{c}(x), x \in \mathcal{O}, \mathcal{O}$ open be a continuous section of the sheaf $\tilde{\mathscr{S}}_{c} . s$ summarizes the local information on the laws as well as on the initial conditions. Continuity means that to each point $x \in \mathcal{O}$ there is a neighborhood $\mathcal{O}_{x}$ of $x$ and a state $\omega_{x} \in \mathscr{S}_{c}\left(\mathcal{O}_{x}\right)$ such that $s_{y}$ is the germ of $\omega_{x}$ at $y$ for all $y \in \mathcal{O}_{x}$. We may choose the neighborhoods $\mathcal{O}_{x}$ to be path connected and such that their intersections are connected. For all $z \in \mathcal{O}_{x} \cap \mathcal{O}_{y}, \omega_{x}$ and $\omega_{y}$ have the same germ at $z$, i.e. they coincide in a neighborhood of $z$. Thus from Theorem $4.1 \omega_{x}$ and $\omega_{y}$ coincide on $\mathcal{O}_{x} \cap \mathcal{O}_{y}$. This is exactly the compatibility condition (4.8).

The preceding attempt to construct a global state from a compatible system of partial states corresponds to the question as to whether a continuous section in $\tilde{\mathscr{S}}_{c}$ gives an element of the presheaf $\mathscr{S}_{c}$. In the case of $\mathscr{S}_{c}$ the answer was not complete due to the mentioned domain problems. Instead of $\mathscr{S}_{c}$ one may consider other presheafs, e.g. the presheaf of local nets of von Neumann algebras without internal symmetries and with a distinguished fully correlated state. In the latter case we have shown that every continuous section over a path connected, simply connected region does correspond to an element of the presheaf.

\section{Summary and Outlook}

Sections I and IV show that basic tenets of the general theory of the relativity, namely general covariance and strict locality, can be incorporated into quantum theory. These sections also give a rough sketch of the frame of such a unified theory.

Many questions remain to be answered before a viable theory along these lines can be proposed. The most important one is to find an explicit charaterization of the germs. In particular, what is their relation to a "Lagrangian"? Further: does the distinction between space-like and time-like directions become established only at scales large compared to the Planck length? In Sect. III we have attempted to obtain some partial information about the germs using the assumption that the theory has a scaling limit. Then each quantum state defines a reduced theory in the tangent space of each point and one of the possibilities is that it thereby defines a metric field. This possibility fits into the picture of an asymptotically free quantum field theory where gravitational effects are included in a semiclassical way. The other interesting possibility is that the tangent space theory has higher symmetry and that the metric evolves only at scales large compared to the Planck length. Nothing has been said here about the mechanism which could lead to a macroscopic metric by a cooperative effect. This needs, of course knowledge about the dynamical laws contained in the germs. Other untouched problems are the rôle of local gauge invariance and of spinor fields. The relation of technical properties of partial states 
used above such as "full correlation" and "tameness" should be better understood in their relation to their behavior under diffeomorphisms and to a precise characterization of local stability of the theory.

There are other aspects within this scenery which have an interest in their own right. Among them is the use of general covariance for the computation of the response of idealized instruments in non-uniform motion as illustrated in an example in Sect. II.

Nothing in the formal structure described depends significantly on the dimension of the underlying manifold. Conceptually, however, unless we choose it as 4-dimensional space-time, it will not fit with the orthodox interpretation of quantum theory, in particular Bohr's emphasis on the necessity of an unambiguous (classical) description of the operational side and the Heisenberg cut.

It is, of course, entirely open whether future theory will develop along these lines or whether the reference to an underlying manifold and strict locality will disappear. Irrespective of the ultimate answer a clarification of the scope of a synthesis between the conventional tenets of general relativity and those of quantum physics is important.

\section{Appendix}

In this appendix we want to describe how one can pass from the operator algebras $\pi_{\omega}(\mathfrak{A}(\mathcal{O}))$ in the GNS representation induced by some state $\omega$ on $\mathfrak{A}$ to von Neumann algebras $\mathfrak{R}_{\omega}(\mathcal{O})$.

Let $\omega$ be a state on $\mathfrak{A}$, i.e. a linear functional on $\mathfrak{A}$ with

$$
\omega(1)=1, \quad \text { and } \quad \omega\left(A^{*} A\right) \geqq 0
$$

for all $A \in \mathfrak{A}$. Then, by the GNS construction, one has a Hilbert space $\mathfrak{H}_{\omega}$, a representation of $\mathfrak{A}$ by operators on some dense subspace $\mathfrak{D}_{\omega}$ of $\mathfrak{S}_{\omega}$, and a unit vector $\Omega_{\omega} \in \mathfrak{H}_{\omega}$, such that

$$
\pi_{\omega}(\mathfrak{Q}) \Omega_{\omega}=\mathfrak{D}_{\omega}, \quad\left(\Omega_{\omega}, \pi_{\omega}(A) \Omega_{\omega}\right)=\omega(A), \quad A \in \mathfrak{A} .
$$

The operator $\pi_{\omega}(A)$ may be unbounded; however, because of

$$
\pi_{\omega}\left(A^{*}\right) \subset \pi_{\omega}(A)^{*}
$$

its adjoint is densely defined, thus $\pi_{\omega}(A)$ is always closable. Let

$$
\pi_{\omega}(A)^{-}=V_{A}\left|\pi_{\omega}(A)\right|
$$

denote the polar decomposition of the closure $\pi_{\omega}(A)^{-}$of $\pi_{\omega}(A)$. Then $\Re_{\omega}(\mathcal{O})$ may be defined as the von Neumann algebra which is generated by $V_{A}$ and the spectral projections of $\left|\pi_{\omega}(A)\right|$ for all $A \in \mathfrak{H}(\mathcal{O})$. $\mathfrak{R}_{\omega}(\mathcal{O})$ is the smallest von Neumann algebra to which all operators $\pi_{\omega}(A)^{-}, A \in \mathfrak{A}(\mathcal{O})$, are affiliated.

We now can turn to the proof of Theorem 3.2. Let $\omega$ be a state on $\mathfrak{A}$ belonging to some primary folium $F$. Then for $\bar{P} \in \mathscr{M}$,

$$
\bigcap_{\sigma_{\in} P} \Re_{\omega}(\mathcal{O})=\{\lambda 1 \mid \lambda \in \mathbb{C}\} .
$$

Now assume that $\omega$ has a scaling limit $\omega_{\bar{P}}$ at $\bar{P}$ with the scaling function $N(\lambda)$ (cf. 
(3.8)). Let for $\hat{f} \in \hat{\mathfrak{A}}, \lambda>0$ sufficiently small,

$$
f_{\lambda}^{(n)}=N(\lambda)^{n} \beta \hat{\alpha}_{\lambda} \hat{f}^{(n)}, \quad n \in \mathbb{Z}_{+},
$$

and define for $f \in \mathfrak{U}$ and $c>0$,

$$
A_{c}(f)=\left(1+c^{2}\left|\pi_{\omega}(f)^{*}\right|^{2}\right)^{-1} \pi_{\omega}(f)^{-} .
$$

We have

$$
\begin{gathered}
\left\|A_{c}(f)\right\| \leqq \frac{1}{2 c} \\
\left\|\left(A_{c}(f)-\pi_{\omega}(f)\right) \bar{\Phi}\right\| \leqq \frac{c}{2}\left\|\pi_{\omega}\left(f^{*} f\right) \bar{\Phi}\right\|, \quad \Phi \in \mathfrak{D}_{\omega}, \\
\left|\left(\bar{\Phi},\left(A_{c}(f)-\pi_{\omega}(f)\right) \bar{\Phi}\right)\right| \leqq \frac{c}{2}\left\|\pi_{\omega}\left(f^{*}\right) \Phi\right\|\left\|\pi_{\omega}(f) \bar{\Phi}\right\|, \quad \bar{\Phi} \in \mathfrak{D}_{\omega}, \\
A_{c}(f) \in \mathfrak{R}_{\omega}(\mathcal{O}) \quad \text { if } \quad f \in \mathfrak{A}(\mathcal{O}) .
\end{gathered}
$$

\section{A.1. Lemma.}

$$
w-\lim _{c \rightarrow 0} w-\lim _{\lambda \rightarrow 0} A_{c}\left(f_{\lambda}\right)=\omega_{\bar{P}}(\hat{f}) 1 .
$$

Proof. Let $\mathcal{O}$ be an arbitrary open neighborhood of $\bar{P}$. Then for $\lambda$ sufficiently small $A_{c}(\lambda) \in \mathfrak{R}_{\omega}(\mathcal{O})$ for all $c>0$. Hence according to Eq. (A.5) the weak limit points of the uniformly bounded sequence $\left(A_{c}(\lambda)\right)_{\lambda \rightarrow 0}$ are multiples of the identity, i.e.

$$
A_{c}\left(f_{\lambda}\right)-\left(\Omega_{\omega}, A_{c}\left(f_{\lambda}\right) \Omega_{\omega}\right) 1 \underset{w}{\longrightarrow} 0, \quad \lambda \rightarrow 0 .
$$

But by inequality (A.8) (iii) these limit points differ from the limit $\omega_{\bar{P}}(\hat{f})$ of the sequence $\left(\Omega_{\omega}, \pi_{\omega}\left(f_{\lambda}\right) \Omega_{\omega}\right)=\omega\left(f_{\lambda}\right), \lambda \rightarrow 0$ at most by $\frac{c}{2} \omega_{\bar{p}}\left(\hat{f}^{*} \hat{f}\right)^{1 / 2} \omega_{\bar{p}}\left(\hat{f}^{*}\right)^{1 / 2}$. In the limit $c \rightarrow 0$ one obtains the statement of the lemma. q.e.d.

It is now easy to find a dense set in $\mathfrak{H}_{\omega}$ for which the scaling limit exists. Namely, let $\mathcal{O}$ be an open neighborhood of $\bar{P}$ and let $B^{\prime} \in \mathfrak{R}_{\omega}(\mathcal{O})^{\prime}$. Then $B^{\prime} \Omega_{\omega}$ is in the domain of definition of $\pi_{\omega}(f)^{-}$for all $f \in \mathfrak{A}(\mathcal{O})$. We have for an arbitrary $f \in \mathfrak{A}$ and $\lambda$ sufficiently small,

$$
\begin{aligned}
\left(B^{\prime} \Omega_{\omega}, \pi_{\omega}\left(f_{\lambda}\right)^{-} B^{\prime} \Omega_{\omega}\right) & =\left(B^{* *} B^{\prime} \Omega_{\omega}, \pi_{\omega}\left(f_{\lambda}\right) \Omega_{\omega}\right) \\
& =\left(B^{\prime *} B^{\prime} \Omega_{\omega},\left(\pi_{\omega}\left(f_{\lambda}\right)-A_{c}\left(f_{\lambda}\right)\right) \Omega_{\omega}\right)+\left(B^{*} B^{\prime} \Omega_{\omega}, A_{c}\left(f_{\lambda}\right) \Omega_{\omega}\right) .
\end{aligned}
$$

In the limit $\lambda \rightarrow 0, c \rightarrow 0$ the first term in the last line of this equation vanishes according to inequality (A.8), the second term converges to $\left\|B^{\prime} \Omega_{\omega}\right\|^{2} \omega_{\bar{P}}(\hat{f})$ according to Lemma A.1. Hence in the state induced by $B^{\prime} \Omega_{\omega}$ the scaling limit exists with the same scaling function $N(\lambda)$ and coincides with $\omega_{\bar{P}}$. From Eq. (A.5) the set $\bigcup_{\mathcal{O} \in \bar{P}} \mathfrak{R}_{\omega}(\mathcal{O})^{\prime} \Omega_{\omega}$ is dense in $\mathfrak{H}_{\omega}$, thus the finite convex combinations of the induced states are a dense subset of the folium $\mathscr{F}$ on which the scaling limit exists and coincides with $\omega_{\bar{P}}$. 


\section{References}

1. Einstein, A.: Äther und Relativitätstheorie. Vortrag Universität Leiden. Berlin, Heidelberg, New York, Springer 1920

2. Ekstein, H.: Presymmetry II. Phys. Rev. 184, 1315 (1969)

3. Avishai, Y., Ekstein, H.: Presymmetry of classical relativistic fields. Phys. Rev. D7, 983 (1973)

4. Borchers, H. J.: On the structure of the algebra of field operators. Nuovo Cimento 24, 214 (1962); Uhlmann, A.: Über die Definition der Quantenfelder nach Wightman und Haag, Wissenschaftl. Zeit. d. KMU Leipzig, 11, Math. Nat. Reihe Heft 2 (1962)

5. Haag, R., Narnhofer, H., Stein, U.: On quantum field theory in gravitational background. Commun. Math. Phys. 94, 219 (1984)

6. Erdelyi, A., Magnus, W., Oberhettinger, F.: Higher transcendental functions. New York, Toronto, London: McGraw-Hill 1953

7. Bell, J. S., Leinaas, J. M.: Electrons as accelerated thermometers. Nucl. Phys. B212, 131 (1983)

8. Bisognano, J. J., Wichmann, E. H.: On the duality condition for a Hermitian scalar field. J. Math. Phys. 16, 985 (1975); On the duality condition for quantum fields. J. Math. Phys. 17, 303 (1976)

9. Reeh, H., Schlieder, S.: Bemerkungen zur Unitäräquivalenz von lorentzinvarianten Feldern. Nuovo Cimento 22, 1051 (1961)

10. Driessler, W.: Duality and absense of locally generated superselection sectors for CCR-type algebras. Commun. Math. Phys. 70, 213 (1979)

11. Roberts, J. E.: Private communication, dated January 1983

12. Fredenhagen, K., Hertel, J.: Local algebras of observables and pointlike localized fields. Commun. Math. Phys. 80, 555 (1981)

13. Dubois-Violette, M.: A Generalization of the classical moment problem on *-algebras with applications to relativistic theory. Commun. Math. Phys. 43, 225 (1976), 54, 151 (1977)

Communicated by R. Haag

Received July 11, 1986 
